\title{
Propuesta de una herramienta informática para agilizar la revisión de instrumentos evaluativos basados en respuestas abiertas
}

\author{
Francisco Velásquez Opazo \\ fvelasquez.opazo@gmail.com \\ https://orcid.org/0000-0002-6462-3480 \\ Juan Pinto Canales \\ https://orcid.org/0000-0001-7435-6470
}

Santiago-Chile

\section{RESUMEN}

La evaluación es una importante etapa del aprendizaje, ya que permite determinar logros y las decisiones que un docente aplique en un futuro a corto o largo plazo. De todos los instrumentos existentes, al menos en el ámbito de una sala de clases, la evaluación de respuesta abierta es una de las más complejas al momento de revisar, ya que no solo se le debe dedicar más tiempo para esta tarea, sino también una comprensión de una respuesta que hasta ahora solo una persona puede lograr; lo que implica que, para este tipo de evaluaciones, no existan softwares al alcance de un típico docente, que le permitan la correspondiente revisión en forma más ágil. Una situación completamente opuesta ocurre con las evaluaciones de selección múltiple. En esta propuesta se muestra un libro de cálculo diseñado en el programa Microsoft Excel, que permite, al menos en parte, cerrar la brecha existente entre las evaluaciones de desarrollo y los softwares disponibles que pueden facilitar su revisión. Esta herramienta informática, llamada HAPRE, requiere que el docente ingrese algunos datos necesarios como porcentaje de exigencia, puntaje de la evaluación, ponderación de los ítems, etc. Luego el docente solo debe dedicarse a ingresar los niveles de logro por pregunta.

Palabras claves: informática; Excel; prueba de desarrollo; evaluación 


\title{
Proposal for a computer tool to speed up the review of open response- based evaluation instruments
}

\begin{abstract}
Evaluation is an important stage of learning, as it enables the identification of achievements and decisions that a teacher will make in the short or long term. Of all existing instruments, at least within a classroom, open response assessment (development of responses) is one of the most complex ones to review, as not only should more time be devoted to this task, but also an understanding of a response that so far only one person can achieve; implying that for such evaluations, there are no software at the reach of a typical teacher, which allows the corresponding revision in a more agile way. A completely opposite situation occurs with multiple-choice evaluations. This proposal shows a workbook designed in the Microsoft Excel program, which allows, at least in part, to close the gap between development evaluations and available software that can facilitate their review. This computer tool, called HAPRE, requires the teacher to enter some necessary data such as percentage of demand, score of the evaluation, weighting of the items, etc. Then the teacher should only focus on entering achievement levels by question.
\end{abstract}

Keywords: computer science; excel; open-ended questions; evaluation

Artículo recibido: 15 enero 2022 Aceptado para publicación: 08 febrero 2022 Correspondencia: fvelasquez.opazo@gmail.com Conflictos de Interés: Ninguna que declarar 


\section{INTRODUCCIÓN}

"Todo proceso evaluativo, destinado a evaluar el aprendizaje de los y las estudiantes debiera cumplir con cinco etapas: Planificación, construcción, administración, análisis y por último certificación”. Juan José Gutiérrez Paredes. (2016). Formación Basada En Competencia "Procedimientos evaluativos y calificativos del desempeño de los estudiantes". p..69)

Sin embargo, la evaluación en cualquiera de sus formas es una necesidad implícita en el proceso de enseñanza-aprendizaje, aunque en algún momento se le puede usar como un instrumento discriminador entre mejores y peores, "lo cierto es que ha evolucionado para ser un instrumento al servicio de los aprendizajes, y no como un fin de éstos, es decir, un proceso continuo, participativo y transparente". Rodríguez Conde, Ma José. (2005). Aplicación de las TIC a la Evaluación de Alumnos Universitarios. Teoría de la Educación. Educación y Cultura en la Sociedad de la Información, (vol. 6, núm. 2). Universidad de Salamanca.

Por cierto, "La evaluación permite valorar los aprendizajes, a la vez que debe permitir mejorarlos”. Fernández, Sonsoles. (2012.) Escuela Oficial de Idiomas de Madrid. Evaluación y Aprendizaje. (intro, 4)

"Lo fundamental de la evaluación, no es hacer evaluación, ni siquiera hacerla bien, Lo fundamental es conocer el papel que desempeña, la función que cumple, saber quién se beneficia de ella y, en definitiva, al servicio de quién se pone”. Ruíz Ruíz, J. M. (2016). Cómo hacer una evaluación de centros educativos (3a. ed.). Madrid, Spain: Narcea Ediciones. Recuperado de.https://elibro.net/es/ereader/iacc/45977?page=17

Se consigna que el párrafo "ni siquiera hacerla bien" no se refiere a un contexto de la forma, sino más bien del fondo del instrumento evaluativo.

Una evaluación correctamente aplicada, no solo sirve para determinar el manejo de ciertos conocimientos y/o habilidades; también se transforma en una valiosa ayuda para el estudiante, ya que permite una adecuada retroalimentación que le informará de sus errores y lo que puede mejorar; los errores también enseñan. A la vez, el docente podrá apreciar el nivel de aprendizaje que tiene sus estudiantes, particularmente sus dificultades, y de esa forma, contar con mejor información para tomar las decisiones para adecuar su metodología, cambiarla por completo, o mantenerla. Es más, "Una adecuada revisión de 
los resultados permitirá determinar si el instrumento evaluativo estaba confeccionado adecuadamente. M. Gabriela Vázquez Olivera. (2016). Problemas y limitaciones del uso de pruebas estandarizadas para la evaluación educativa en México. (p. 2, 22)

"Existen numerosos argumentos a favor del establecimiento de sistemas de evaluación educativa sólidos y confiables, los cuales son de uso común, por lo que no es necesario incluir aquí más que un breve resumen: Toma de decisiones, rendición de cuentas, distribución de incentivos y participación social y elección”. Universidad Abierta de México. (2018). Evaluación educativa: Condiciones, procesos e implicancias en la $\begin{array}{lllll}\text { enseñanza } & \text { y } & \text { el } & \text { aprendizaje. } & \text { Recuperado }\end{array}$ de.https://revista.universidadabierta.edu.mx/2019/11/29/evaluacion-educativacondiciones-procesos-e-implicaciones-en-la-ensenanza-y-el-aprendizaje/

"Las pruebas de opción múltiple pertenecen al grupo de pruebas estructuradas y de tipo escrito. Son preguntas (enunciados o base del reactivo) con varias posibles respuestas (opciones) de las cuales una es la correcta y las restantes (distractores) son verosímiles, o de las que todas son parcialmente correctas, pero sólo una de ellas es la más apropiada; en el primer caso, son llamadas de respuesta correcta y, en el segundo, de respuesta óptima”. Calidad Académica. Opción múltiple. Extraído de. http://sitios.itesm.mx/va/calidadacademica/files/opcion.pdf

Es muy difícil indicar cuáles son los tipos de instrumentos evaluativos más utilizados en un colegio, diferentes profesores utilizan diferentes metodologías e incluso también depende del PEI del establecimiento, el que puede dar más libertad a los profesores o restringirlos a utilizar cierto instrumento.

Es posible establecer 2 grandes grupos de evaluaciones, que son de respuesta cerrada o respuesta abierta, ambas con sus diferentes potencialidades y desventajas. Mientras las de respuesta cerrada son muy acotadas en lo que se busca medir y son muy rápidas de revisar, representan mucho tiempo para construir el instrumento adecuadamente y son solo útiles para medir niveles básicos de aprendizaje. Las evaluaciones de respuesta abierta literalmente son lo opuesto Ya que, en los colegios, normalmente se atiende a muchos estudiantes por curso, el instrumento de respuesta cerrada es más utilizado para obtener resultados y calificaciones en un corto tiempo. Lo anterior no es impedimento para aplicar evaluaciones de respuesta abierta, pero son menos utilizados por el obvio problema de tiempo que demanda su revisión. José Ma Arribas Estebaranz. Juan Carlos Manrique 
Arribas y Belén Tabernero Sánchez. (2014). Instrumentos de evaluación utilizados en la formación inicial del profesorado y su coherencia para el desarrollo de competencias profesionales en los estudiantes: visión del alumnado, egresados y profesorado.

Las prácticas evaluativas corresponden a las acciones continuas y sistemáticas que realizan los docentes para recoger información relevante del estado y del proceso de aprendizaje de los estudiantes, que le permite formar juicios de valor a partir de los cuales toman decisiones para la mejora de los procesos de enseñanza y de aprendizaje. Curriculum Nacional.

Extraído de.https://www.curriculumnacional.cl/portal/Tipo/70896:

Dicho lo anterior, se enfatiza que, en las evaluaciones de preguntas cerradas, al estudiante no se informa cómo logró llegar a una respuesta errada, no es el caso de las preguntas abiertas el cual el estudiante visualiza sus errores y es capaz de desarrollar su propio feedback.

Sin embargo, en ambos instrumentos el docente ocupa un gran tiempo pedagógico, para revisar y calificar dichas evaluaciones. Pero es importante destacar que las preguntas de tipo cerradas implican menos tiempo pedagógico de revisión, siempre y cuando el docente cuente con una plantilla que le permita contar las respuestas correctas; o cuente con algún escáner que le permita ingresar estas respuestas al sistema de herramienta digital.

Por el contrario, las preguntas de respuestas abiertas son extensas en el uso de tiempo pedagógico, por el simple hecho de intercalar argumentos e indicadores que estén dentro de las respuestas a dicha preguntas abiertas, el docente utiliza más tiempo por el simple hecho de hacer lectura de las respuestas y analizarlas. Además, podemos añadir que los resultados pueden estar con demora por el simple hecho que nuestros estudiantes utilizan muy mala ortografía y una mala redacción. Dicho lo anterior se puede evidenciar a través de la Universidad del Zulia. Nivel de redacción de textos académicos de estudiantes ingresantes a la universidad Año 33, No. 84 (2017): 791-817 ISSN 1012-1587/ISSNe: 2477-9385

El desarrollo de la informática ha permitido crear numerosas herramientas digitales para facilitar la enseñanza-aprendizaje. Existe un avance similar para ayudar al docente al momento de calificar las evaluaciones y obtener un valioso análisis estadístico que es útil para la retroalimentación. En sitios web como https://www.educaciontrespuntocero.com/recursos/herramientas-para-corregir- 
examenes/, se pueden encontrar diferentes herramientas digitales que permiten crear y revisar evaluaciones. Pero para que sean efectivos, deben ser de respuesta cerrada para que el programa pueda hacer el trabajo de revisión. No existe un software, al menos, uno que esté al alcance de un docente, que sea capaz de leer una respuesta escrita por una persona, comprender dicha respuesta y determinar si es correcta o no, en base a una pauta dada. Al respecto, hay incluso aplicaciones para celulares, que permiten escanear un formulario de respuestas de selección múltiple, y proporcionar una revisión ágil de dicha evaluación.

Así, existen numerosas herramientas informáticas que permiten la creación y posterior revisión automática para evaluaciones de respuesta cerrada, pero ni una con esas características para evaluaciones de respuesta abierta; estableciéndose una gran brecha digital entre estos tipos de evaluaciones.

El uso de las TIC reviste una especial importancia en el mundo actual, lo que implica que los docentes deben modernizarse sobre sus metodologías para incorporar herramientas digitales en su quehacer y de ser necesario, según lo planteado por Lanuza, Rodríguez y Saavedra. (2018). Uso y aplicación de las TIC en el proceso de enseñanza-aprendizaje. Debe existir la adecuada capacitación, aceptando las ventajas que ofrece el manejo de estas herramientas. Según este estudio, tanto docentes como estudiantes tienen un manejo similar en las TIC; diferenciándose en el uso que le dan a la información obtenida en medios electrónicos, donde los estudiantes llevan la delantera. Si bien docentes y estudiantes valoran el uso de las TIC como apoyo pedagógico, los últimos las valoran y utilizan más; sin desmerecer que ambos grupos consideran ser proactivos respecto a su uso y aplicación. Por lo tanto, si se trata de algo que contribuya al proceso de enseñanzaaprendizaje, el uso de una herramienta informática será apreciado.

El uso del programa Microsoft-Excel por parte de los docentes está ampliamente documentado. Particularmente en se documenta el uso para varias instancias escolares, como asistencia, accesibilidad y entrega de calificaciones, llevar un seguimiento al cuantificar los aprendizajes, etc. Excel tiene una serie de ventajas, su uso es muy amplio, su manejo es fácil de aprender, existen muchos tutoriales publicados en el sitio www.youtube.com.

En la tesis de Velásquez Opazo, Francisco Javier, and Juan Guerrero Núñez. "Procesamiento de datos utilizando una herramienta informática para facilitar la 
corrección de evaluaciones en clases.” Tesis Universidad de Santiago de Chile, (2012). Se propuso un libro de cálculo basado exclusivamente en el uso de Excel, para permitir no solo la revisión de evaluaciones de selección múltiple, sino además obtener una información estadística de fácil visualización para el docente, quién casi en tiempo real podía ver información como porcentajes de aprobación por pregunta, factores de discriminación, diferentes escalas de calificaciones, y otros ajustes que permitían ajustar la evaluación y su posterior calificación, a la realidad del grupo-curso.

Constantemente, las funciones de cálculo de Excel son utilizadas por los docentes, ya sea individual o grupalmente, para llevar registro de alguna actividad y a la vez, ordenar diferentes datos para que su lectura sea entendible.

Las evaluaciones de respuesta cerrada han sido grandemente beneficiadas por las herramientas informáticas existentes, que permiten mayor rapidez en la revisión con su correspondiente calificación; a la vez que el análisis de los datos que realizan los mismos softwares de revisión, permiten una mejor retroalimentación tanto para el estudiante como para el docente. Todo lo anterior en un tiempo muy corto comparado con sus contrapartes, las evaluaciones de respuesta abierta.

En este trabajo se propone una herramienta informática que pueda, al menos, disminuir la brecha descrita anteriormente. Se diseñó un libro de cálculo basado en la propuesta de Velásquez Opazo, Francisco Javier, and Juan Guerrero Núñez. "Procesamiento de datos utilizando una herramienta informática para facilitar la corrección de evaluaciones en clases ." Tesis Universidad de Santiago de Chile, (2012), Llamado EPSEMU (Evaluador de Pruebas de Selección Múltiple); con varias modificaciones para permitir el ingreso de los datos que se necesitan para la revisión de evaluaciones de respuesta abierta. En este caso, al libro de cálculo resultante se le llamó HAPRE (Herramienta Administrativa Para Revisión de Evaluaciones).

La automatización obtenida es parcial, ya que leer, interpretar y asignar puntajes de logro por respuesta, aún es algo que solo el docente puede hacer. La ayuda que entrega el libro de cálculo propuesto es asumir el cálculo de puntajes y el análisis de datos sobre la revisión de las evaluaciones, de manera que proporcione al docente, información casi en tiempo real, optimizando el esfuerzo y el tiempo del docente en la obtención de calificaciones y la información necesaria para la retroalimentación correspondiente. Este último aspecto puede ser el más valioso, ya que el software permite mostrar los resultados 
con diferentes gráficos, tablas y otras formas de visualización que demandan un esfuerzo y tiempo poco viables, si se hicieran en forma manual, pero que son muy valoradas cuando se tienen disponibles, ya que así el docente cuenta con datos numéricos para cualquier decisión que haga respecto a su metodología y el aprendizaje correspondiente. El libro de cálculo propuesto, al igual que el de Velásquez, (2012). está íntegramente hecho en el programa Microsoft Excel, un software muy conveniente por razones descritas anteriormente: amplio uso, instalado por defecto en casi cada computador que un docente utilice, y cuyo aprendizaje puede ser incluso autodidacta; haciendo que esta plataforma sea ideal para el manejo requerido. Es fácilmente modificable de acuerdo con los requerimientos del docente y/o de una situación en particular, haciendo que el libro de cálculo propuesto sea muy flexible en su uso.

\section{METODOLOGÍA}

El libro de cálculo propuesto se basa en uno que se propuso en la tesis "Procesamiento De Datos Utilizando Una Herramienta Informática Para Facilitar La Corrección De Evaluaciones En Clases" (Velásquez, 2013). En dicho trabajo, se describe un libro de cálculo llamado EPSEMU para la revisión de evaluaciones de selección múltiple; en el cual, el docente debía ingresar las respuestas de los estudiantes, y el libro realizaba los cálculos de notas y entregaba otras informaciones estadísticas de interés. Muy similar a otros softwares comerciales, aunque EPSEMU ofrece la ventaja de la personalización y una plataforma informática utilizada por muchos docentes.

Para crear un libro de cálculo que permita la revisión de evaluaciones de respuesta abierta, se conservaron los algoritmos para calcular las calificaciones según el puntaje obtenido y el porcentaje de exigencia, ya que las fórmulas matemáticas implicadas son las mismas; teniéndose que cambiar el ingreso de puntajes y agregar informaciones gráficas para evaluar objetivos o habilidades específicas. A este nuevo libro de cálculo se le ha denominado HAPRE (Herramienta Administrativa Para Revisión de Evaluaciones).

\section{REVISIÓN}

Esta parte aún debe seguir siendo realizada por el profesor, ya que no existe una inteligencia artificial que pueda leer, comprender y asignar un determinado puntaje a una respuesta abierta; de forma similar a como lo haría una persona

En todos los casos, la revisión requiere de una rúbrica de comparación para determinar el puntaje más adecuado, basado en ciertos criterios que el docente determina según la 
pregunta. Para usar mejor el HAPRE, se recomienda que las preguntas sean de una respuesta breve y precisa.

Si se opta por respuestas más complejas, se recomienda dividir la revisión de la respuesta, en secciones más pequeñas. Para el caso de esta propuesta, se probó la revisión de algunas evaluaciones con una rúbrica que evaluara los siguientes aspectos de cada respuesta:

- Conceptos claves: son palabras o pequeñas frases, los que deben estar presentes en una respuesta que sí se ha entendido completamente, y su presencia es necesaria para una respuesta totalmente correcta.

- Redacción: la respuesta debe ser adecuada para la pregunta hecha, que trate de responder la interrogante. Los conceptos claves deben estar relatados en un orden lógico y entendible. La respuesta también debe ser lo más concisa posible

- Ortografía: depende de la materia evaluada y del criterio del profesor. Existen fallas ortográficas son esperables por el nivel que cursa el estudiante y el concepto empleado, mientras otras son muy básicas y definitivamente un determinado estudiante no debería cometerlas, cualquiera sea la asignatura evaluada.

A cada uno de estos ítems, se le puede asignar diferentes ponderaciones, cómo el siguiente ejemplo:

¿Por qué una de las hebras del ADN se sintetiza en forma discontinua? (1)

\begin{tabular}{|l|l|}
\hline Puntaje & $\begin{array}{l}\text { Rúbrica: } \\
\text { Concepto claves: orientación contraria, giro, síntesis de fragmento. }\end{array}$ \\
\hline $50 \%$ & Todos los conceptos claves \\
\hline $40 \%$ & $\begin{array}{l}\text { Redacción adecuada (respuesta entendible) y signos de puntuación } \\
\text { apropiados. }\end{array}$ \\
\hline $10 \%$ & Buena ortografía \\
\hline
\end{tabular}

Fuente: Autoría

El docente debe determinar la ponderación a cada aspecto evaluado por pregunta; para este caso, se han elegido de 3 a 4 . Una vez establecida la ponderación, se propone evitar que el docente deba decidir directamente el puntaje de la respuesta, sin o que, por cada aspecto, solo deba pensar básicamente en 4 niveles de logro:

- 4: Nivel óptimo de logro para el aspecto evaluado (100\%).

- 3: Nivel aceptable de logro para el aspecto evaluado (75\%).

- 2: Solo se alcanza la mitad de logro para el aspecto evaluado (50\%).

- 1: El nivel de logro para este nivel es mínimo (25\%).

- 0 : No hay respuesta para este aspecto. 
Es decir, si se evalúa un aspecto de la respuesta, como conceptos claves, el docente solo debe pensar de 0 a 4, de acuerdo con el nivel de logro explicado arriba, no el puntaje con el que valora dicho ítem a revisar. Se aplica el mismo criterio para la revisión de cualquier otro aspecto. De esta forma se evita que, al valorar la respuesta directamente con un puntaje dado, el docente tenga que discriminar entre 0,9 y 0,$8 ; 0,5$ y 0,$6 ; 0,3$ y 0,2 ; etc. Se logra dar mayor simplicidad a la revisión.

Parecen muchos aspectos a tener presente, pero HAPRE simplifica esa tarea. Una de las pestañas del HAPRE se denomina "Molde", en la que hay espacios como el que se muestra a continuación:
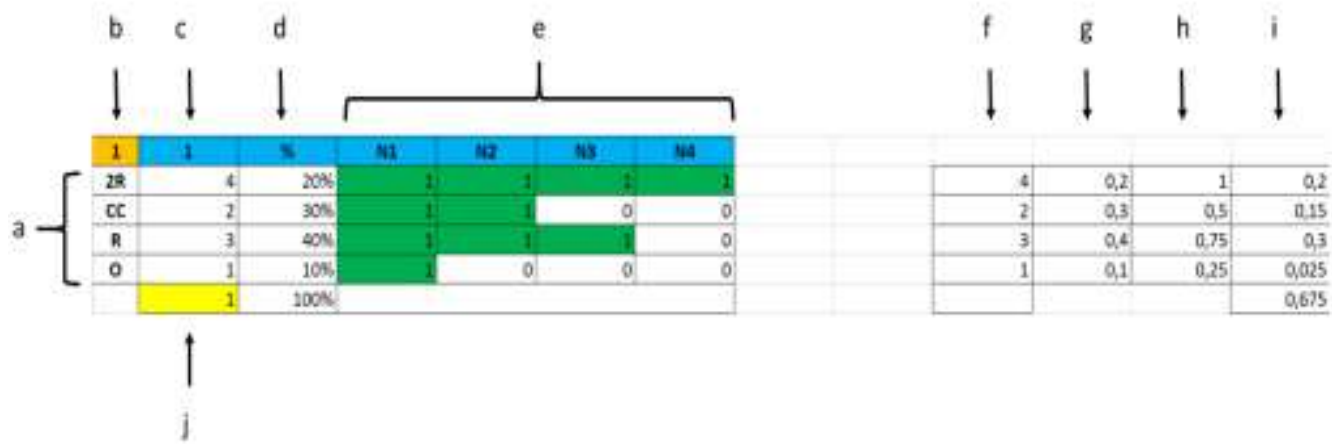

Tabla.1: Sección de la hoja del HAPRE, llamada "molde". En esta sección, el docente ingresa los niveles de logro que el docente asigna, según la calidad de la respuesta del estudiante. Hay parámetros que deben ser ingresados previamente a la revisión. Otros, son calculados automáticamente.

a) Estas 4 casillas de esta columna corresponden a los aspectos evaluados de una pregunta.

b) Esta casilla corresponde al $\mathrm{N}^{\circ}$ de pregunta.

c) La casilla de arriba corresponde al puntaje de la pregunta. En las 4 casillas abajo se ingresa el nivel de logro por cada aspecto evaluado (1, 2, 3 o 4). Estos son los datos que el docente digita cuando ya está ejecutando la revisión de la evaluación.

d) Ponderación. La casilla de arriba ya está señalada con “\%”. En las 4 de abajo, se deben ingresar los porcentajes con que se pondera cada aspecto. HAPRE suma los porcentajes e indica el resultado en la última casilla (en este ejemplo, se muestran los porcentajes de ponderación asignados, la suma de estos se calcula en la casilla de abajo, indicando $100 \%$.

e) Los niveles de logro. HAPRE llena estas casillas según el puntaje ingresado en c). 
f) Los puntajes ingresados en c), se multiplican por el valor de 1 en la casilla j).

g) Los ponderales asignados por cada aspecto, se multiplican cada uno por el puntaje asignado de la pregunta en cuestión, que está en la casilla superior de la columna c).

h) Se dividen por 4 los valores de la columna f. Es por 4 porque son 4 niveles de logro.

i) Se multiplican los valores de la columna g por los de la columna $\mathrm{h}$. Estos son los puntajes individuales por cada aspecto evaluado, según el nivel logro evaluado, considerando también la ponderación asignada y el puntaje total de la pregunta. La suma se muestra en la última casilla de esta columna.

j) Está asignado el valor de "1" por defecto, pudiéndose cambiar a 0. Se agregó esta opción en caso de que también el docente opté por penalizar la copia textual de internet, en cuyo caso debe cambiar el valor de " 1 " a " 0 ", de esa forma, todos los puntajes anteriores, se anulan y aparece una barra en rojo debajo de cada nivel evaluado, según lo muestra el ejemplo de la figura 4.

Los datos en a, b, c y d son determinados por el docente y deben ser ingresados manualmente antes de iniciar la revisión de la evaluación. El resto; HAPRE hace los cálculos automáticamente.

Los datos en a, b y d, el docente debe ingresarlos antes de iniciar la evaluación. Los de la columna c (casillas debajo del puntaje de la pregunta), se ingresan durante la revisión.

\section{La hoja "Molde" se ve según la siguiente figura:}

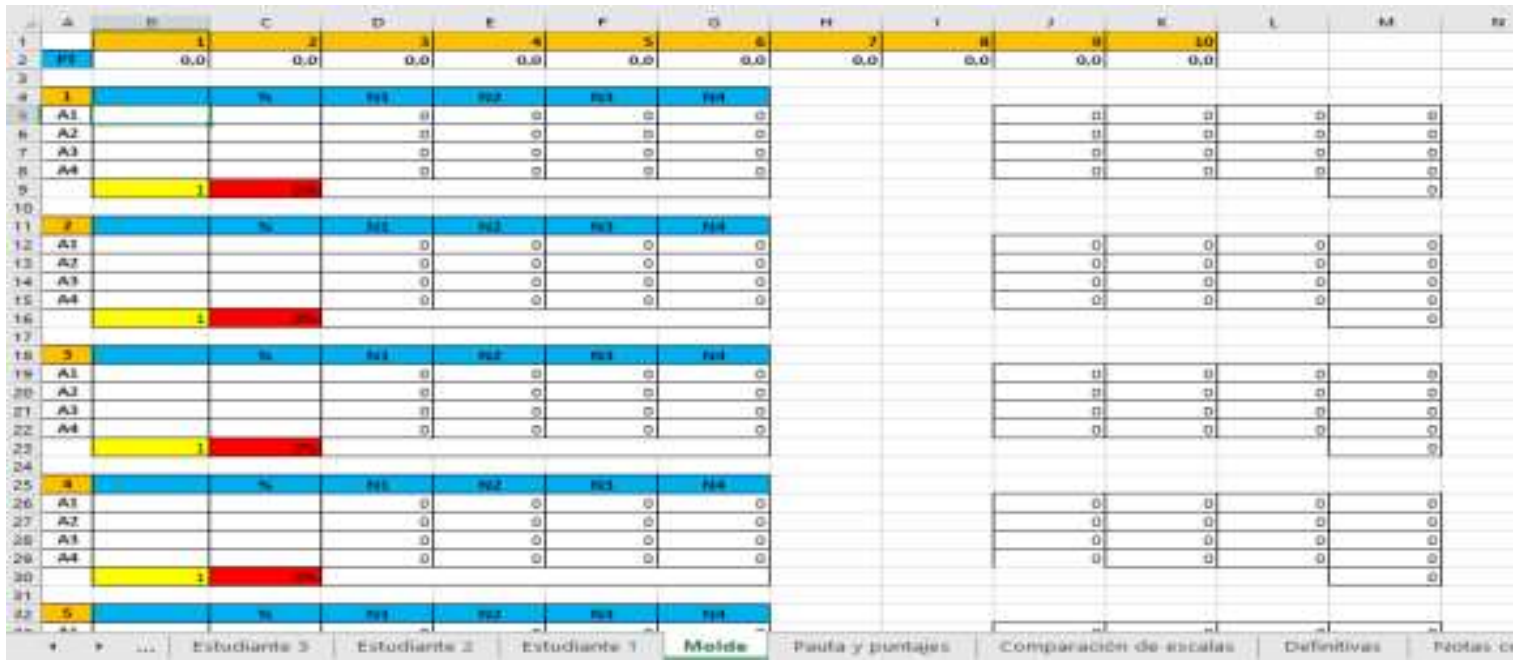

Tabla.2: En la hoja "Molde" existen 10 secciones para la revisión de preguntas. Se pueden agregar más, aunque no es recomendable si se trata de una prueba de respuesta abierta. Las 2 filas superiores corresponden al Reglón resumen; en la fila de arriba, aparece el número de pregunta; en la fila de abajo, los puntajes parciales obtenidos. 
En esta hoja del HAPRE, se determinan las especificaciones de revisión de la evaluación, como los aspectos a evaluar de cada pregunta y su ponderación correspondiente, además de su puntaje correspondiente. Se muestran 4 aspectos máximos a evaluar por pregunta; aunque pueden ser menos, en cuyo caso lo que se recomienda el ocultar los reglones que no se utilizaran. Al terminar de ajustar la hoja "molde" para su uso, se debe ver como lo muestra la siguiente figura:

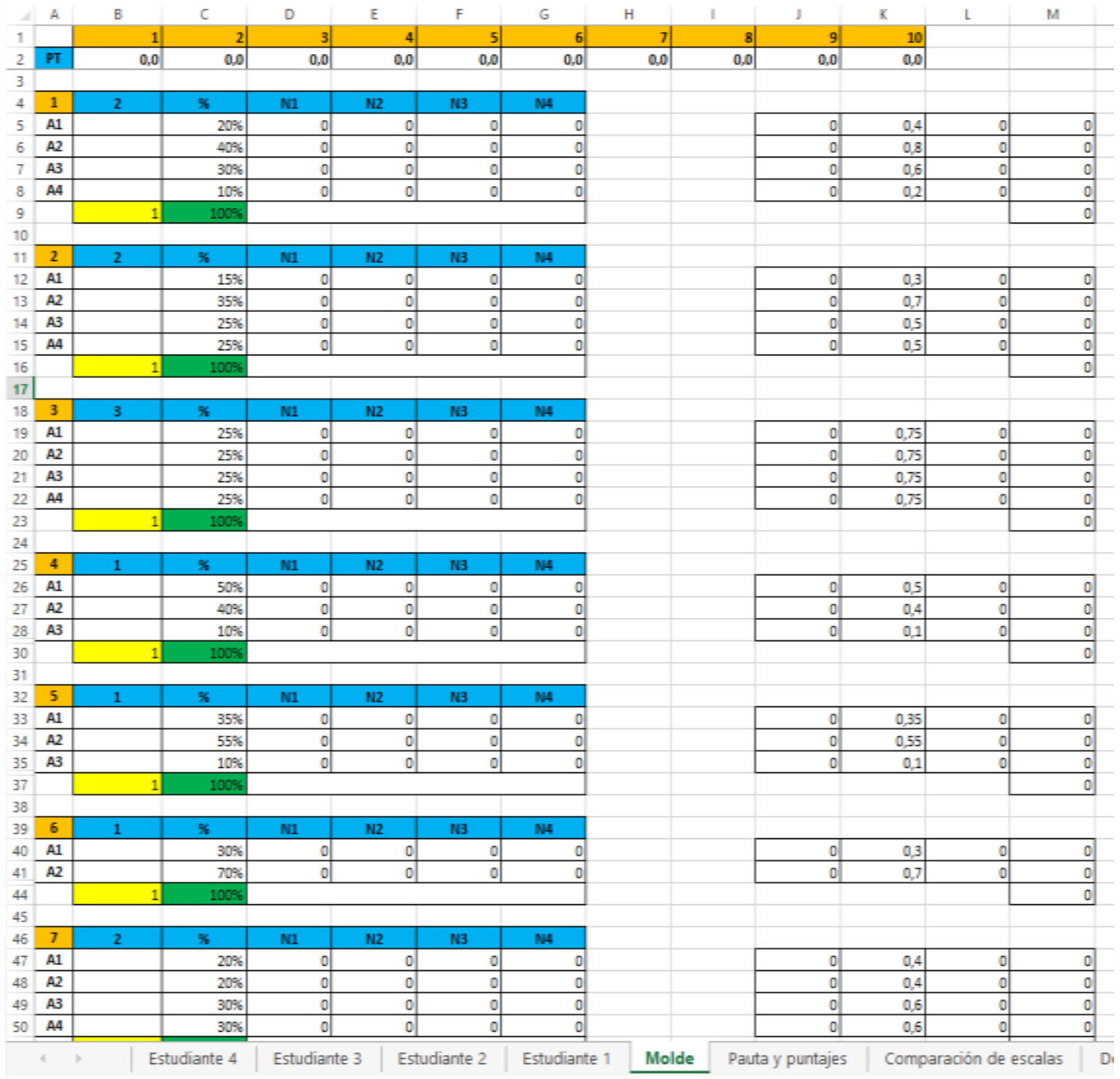

Tabla.3: Hoja "Molde" ajustada y lista para ser utilizada en la revisión de una evaluación. Los aspectos para evaluar por pregunta están indicados como A1, A2, A3 y A4. En algunas preguntas se consideran 4 aspectos a evaluar (1, 2 y 3); otras, solo 3 (4 y 5); o solo 2 (6). A cada aspecto se le ha asignado una ponderación individual que en total debe sumar $100 \%$, haciendo que la casilla al final de esa columna cambie a color verde. Cada pregunta ha sido numerada y tiene su respectivo puntaje asignado. 
Una vez lista, la hoja "Molde" debe permanecer sin cambios. Esta hoja entera debe ser copiada, y la respectiva copia (que Excel mostrará como "molde (2)") debe recibir el nombre que permita identificar al estudiante.

Desde ahí, el docente ya puede empezar la revisión.

Ya no hay que preocuparse por el puntaje de cada aspecto ni del puntaje de cada pregunta, solo debe tener presente, de acuerdo con su rúbrica de comparación, el nivel de logro respectivo e ingresarlo. Al hacerlo mediante el teclado numérico, incluso se puede prescindir de mirarlo, ya que las teclas para ingresar estos datos son fácilmente diferenciables por el tacto. La siguiente figura muestra cómo queda una hoja de cálculo al terminar la revisión de la evaluación de un estudiante.

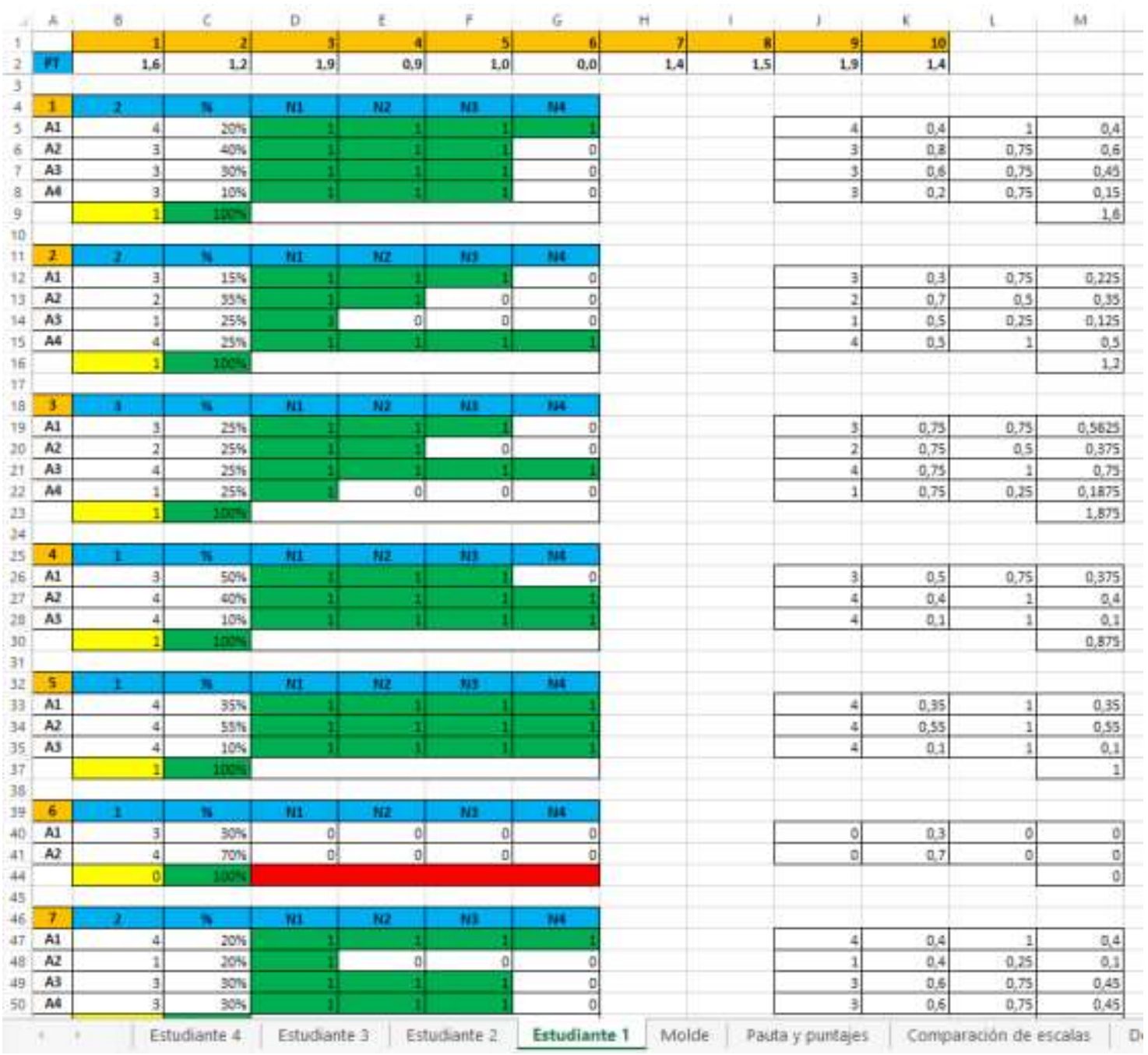

Tabla.4: Hoja "Estudiante 1" que ya contiene los datos de la revisión de su respectiva evaluación. Para cada aspecto evaluado se ha ingresado un valor respectivo entre 1 y 4 , según lo descrito en la letra c de la figura 1. El docente solo se dedica a ingresar los niveles 
de logro, mientras el HAPRE hace el resto de los cálculos para determinar el puntaje respectivo. Todos los puntajes calculados se muestran en la fila "PT".

Nótese como resulta la revisión de la pregunta 6 , cuando el parámetro que está señalado en la letra $\mathrm{j}$ en la figura 1 , cambia a 0 ; todos los puntajes se anulan y aparece una barra roja.

El resultado de esta hoja individualiza la revisión de cada estudiante y puede ser utilizada para la retroalimentación respectiva. Con esta tabla, el estudiante puede ver en qué aspecto de sus respuestas respondió mejor o peor.

Análisis del grupo-curso

La siguiente hoja del HAPRE es donde se ingresan los puntajes respectivos para un análisis del grupo-curso en general, la que se denomina "Pautas y puntajes". En esta hoja también se deben ingresar algunos datos previos a la revisión de la evaluación respectiva, para que el HAPRE pueda hacer los cálculos respectivos y posteriormente entregar la información de interés para el docente y el estudiante. Un ejemplo de la hoja "Pautas y puntajes" lista para ser utilizada, se muestra en las siguientes figuras.

\begin{tabular}{|c|c|c|c|c|c|c|c|c|c|c|c|c|c|c|c|c|}
\hline 4 & & B & c & D & E & F & G & H & 1 & 1 & $x$ & L & M & N & 0 & $p$ \\
\hline 1 & & Aprobación & & & & & & & & & & & & & & \\
\hline 2 & & $N^{2}$ pregunts & & & & & & & & & & & & & & \\
\hline 3 & & Tipo & & & & & & & & & & & & & & \\
\hline 4 & & Habilidad & & & & & & & & & & & & & & \\
\hline 5 & & Puntale total & & & & & & & & & & & & & & \\
\hline 6 & a & Puntaio ajuatado - & $\theta$ & न & 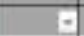 & $\theta$ & F & F & - & - & F & न. & F & $\vec{\theta}$ & 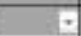 & \\
\hline 7 & 1 & Estudiante 1 & 0.0 & & & & & & & & & & & & & \\
\hline 8 & 2 & Estudiante 2 & 00 & & & & & & & & & & & & & \\
\hline 9 & 3 & Estudiante 3 & 00 & & & & & & & & & & & & & \\
\hline 10. & 4 & Estudiante 4 & 00 & & & & & & & & & & & & & \\
\hline 11 & 5 & Estudiante 5 & 0,0 & & & & & & & & & & & & & \\
\hline 12 & 6 & Estudiante 6 & 00 & & & & & & & & & & & & & \\
\hline 13 & 7 & Estudiante 7 & 0.0 & & & & & & & & & & & & & \\
\hline 14 & 8 & Estudiante 8 & 00 & & & & & & & & & & & & & \\
\hline 15 & 9 & Estudiante 9 & 00 & & & & & & & & & & & & & \\
\hline 16 & 10 & Estudiante 10 & 00 & & & & & & & & & & & & & \\
\hline 17 & 11 & Estudiante 11 & 0,0 & & & & & & & & & & & & & \\
\hline 18 & 12 & Estudiante 12 & 00 & & & & & & & & & & & & & \\
\hline 19 & 13 & Estudiante 13 & 0.0 & & & & & & & & & & & & & \\
\hline 20 & 14 & Estudiante 14 & 00 & & & & & & & & & & & & & \\
\hline 21 & 15 & Estudiante 15 & 0.0 & & & & & & & & & & & & & \\
\hline \multirow{2}{*}{22} & & 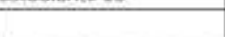 & & & & & & & & & & & & & & \\
\hline & , & H. Iftodurte 3 . & & fante? & Woide & \multicolumn{2}{|c|}{ Pesturpentajen. } & mparsosion & Eecoles & Defint: & & sconpartes & $=1000$ & \multicolumn{3}{|c|}{ 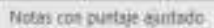 } \\
\hline
\end{tabular}

Tabla.5: Parte superior de la hoja "Pautas y puntajes". En este ejemplo se ha planteado una evaluación de 10 preguntas y un curso de 15 estudiantes. La hoja en sí, tiene espacio para 30 preguntas y 50 estudiantes. El docente debe ingresar previamente: La lista del grupo-curso en las casillas debajo de donde dice "Puntaje ajustado"; en este ejemplo se muestran como Estudiante 1, Estudiante 2, etc. 
El número de pregunta, en la fila señalada como " $\mathrm{N}^{\mathrm{o}}$ pregunta"

La fila indicada como "Tipo" es para que el docente ingrese en una letra o palabra, el tipo de pregunta planteada. Para este ejemplo, la " $\mathrm{t}$ " quiere decir teórica, la "c" es por cálculo, y la "d" es por definición. En este caso, dicha marca queda a criterio del docente y no influye en los cálculos que hace el HAPRE, pero para la retroalimentación, sirve para que el estudiante tenga una idea del tipo de respuesta solicitado.

En la fila de "Habilidad" se ingresa la relación de la pregunta con el contenido y/o habilidad evaluada, la que aparece señalada en la parte inferior de la hoja (Fig-6).

En la fila "Puntaje total", la primera casilla a continuación muestra la suma de todos los puntajes individuales por pregunta. Mientras en esa primera casilla se suman los puntajes en forma autónoma, bajo cada pregunta señalada, se debe ingresar el puntaje individual correspondiente, el que debe coincidir con los puntajes señalados en la hoja "Molde".

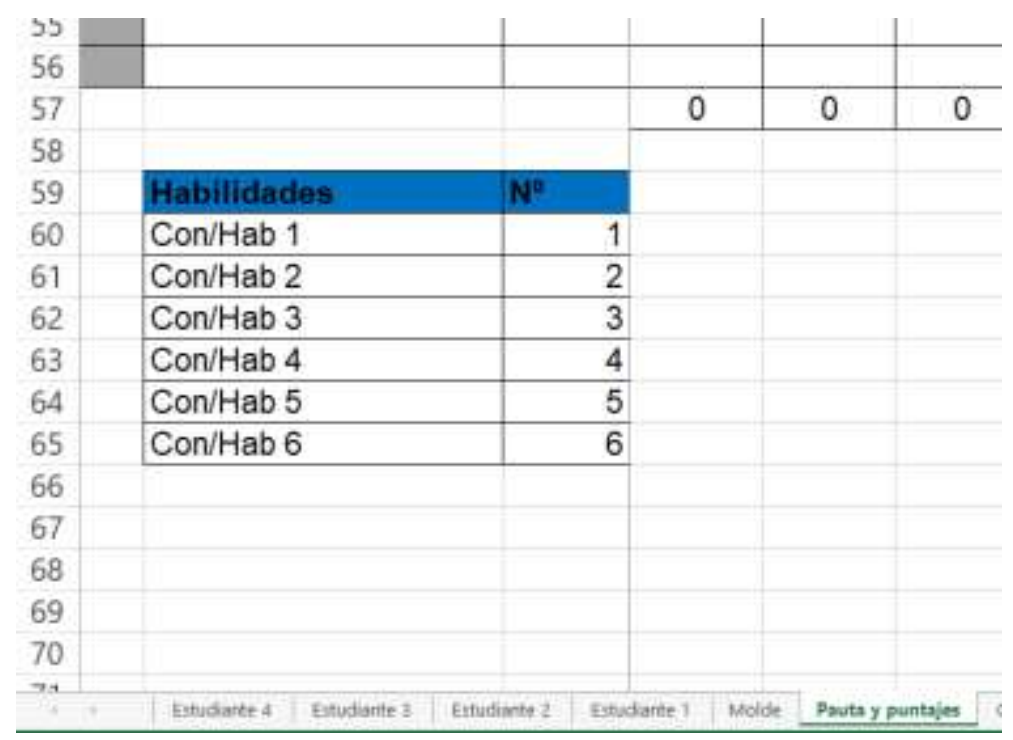

Tabla.6: Parte inferior de la hoja "Pautas y puntajes" que es para determinar los contenidos y/o habilidades a los que se refiere cada pregunta de la evaluación. Hay espacio para 6 de estos ítems. En la columna "Habilidades", se ingresa una palabra que señale en contenido y/o habilidad evaluada en una determinada pregunta. En la columna "N" " se le asigna un valor numérico a dicho contenido/habilidad. De esta forma, el HAPRE puede mostrar la información gráfica respecto al nivel de aprobación por contenido/habilidad evaluada.

Salvo la excepción señalada en la primera casilla de la fila "Puntaje total", los datos indicados deben ser ingresados por el docente. 


\begin{tabular}{|c|c|c|c|c|c|c|c|c|c|c|c|c|c|c|c|c|c|c|}
\hline \multicolumn{6}{|c|}{ 日 $3: 2$} & \multicolumn{8}{|c|}{ 450Natable - hate } & \multicolumn{3}{|c|}{ 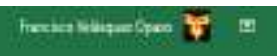 } & \multirow{2}{*}{\multicolumn{2}{|c|}{ 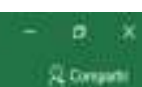 }} \\
\hline \multicolumn{2}{|c|}{ Anten } & wean & \multicolumn{2}{|c|}{ 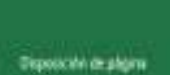 } & Brous: & \multirow[t]{2}{*}{ Ores: } & \multirow[t]{2}{*}{ Exise } & \multirow[t]{2}{*}{$y \mathrm{~m}$} & \multicolumn{5}{|c|}{ Q.porsentans? } & & & & & \\
\hline M & & $-1 \times 1 \times$ & A & & & & & & & & & & & & & & & - \\
\hline 4 & A & \multicolumn{2}{|l|}{8} & c & D & E & F & G & $\mathrm{H}$ & 1 & 1 & k & L & M & $\mathrm{AH}$ & A! & AI & \\
\hline 1 & & \multicolumn{3}{|l|}{ Rprabacon } & & & & & & & & & & & & & & \\
\hline 2 & & \multicolumn{3}{|l|}{ N' preguints } & 1 & 2 & 3 & 4 & 5 & 6 & 7 & 8 & $\theta$ & 10 & & Max prm total & & \\
\hline 3 & & \multicolumn{3}{|l|}{ Tipe } & 1 & $\mathrm{t}$ & E & if & it & d & $t$ & $\mathrm{t}$ & c & $\mathrm{c}$ & & Max prm zis & & \\
\hline 4 & & \multicolumn{3}{|l|}{ Habĩudad } & 1 & $t$ & 2 & 2 & 9 & 3 & 4 & 4 & 5 & 6 & $N^{2}$ dates & Max individual & & \\
\hline 5 & & \multicolumn{2}{|l|}{ Puntaje total } & 18 & 2 & 2 & 3 & $t$ & 1 & 1 & 2 & 2 & 2 & 2 & 10 & & & \\
\hline 5 & 2 & \multicolumn{2}{|l|}{ Puntaie ajustado } & F & $\theta$ & 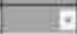 & $\theta$ & & f & $\theta$ & $\theta$ & $\theta$ & $\theta$ & F & $\overrightarrow{7}$ & Nota max & \multicolumn{2}{|l|}{ Thexpener } \\
\hline 7 & 1 & \multicolumn{2}{|l|}{ Estudiante 1} & 0,0 & & & & & & & & & & & 0 & 7) 7,0 & 60,005 & \\
\hline 8 & 2 & \multicolumn{2}{|l|}{ Estudiante 2} & 0,0 & & & & & & & & & & & 0 & 7,0 & 60,005 & \\
\hline 9 & 3 & \multicolumn{2}{|l|}{ Estudiante 3} & 0,0 & & & & & & & & & & & 0 & 7,0 & 60,05 & \\
\hline 10 & 4 & \multicolumn{2}{|l|}{ Estudiamte 4} & 0,0 & & & & & & & & & & & 0 & 7,0 & 60,05 & \\
\hline 11 & 5 & \multicolumn{2}{|l|}{ Estudiame 5} & 0,0 & & & & & & & & & & & 0 & 7,0 & 60,000 & \\
\hline 12 & 6 & Estudiante $\bar{\sigma}$ & & 0,0 & & & & & & & & & & & 0 & 7,0 & $60,0 \%$ & \\
\hline 13 & 7 & Estudiante 7 & & 0,0 & & & & & & & & & & & 0 & 7,0 & 60,035 & \\
\hline 14 & II & Estudiante 8 & & 0,0 & & & & & & & & & & & 0 & 7,0 & 60,03 & \\
\hline 15 & 3 & Estudiante 9 & & 0,0 & & & & & & & & & & & 0 & 7,0 & 60,005 & \\
\hline 16 & 10 & Estudiante 10 & & 0,0 & & & & & & & & & & & 0 & 7,0 & 60,095 & \\
\hline 17 & 11 & Estudiante 11 & & 0,0 & & & & & & & & & & & 0 & 7,0 & 60,05 & \\
\hline 18 & 12 & Estudiante 12 & & 0,0 & & & & & & & & & & & 0 & 7,0 & 60,005 & \\
\hline 19 & 13 & Estudiame 13 & & 0,0 & & & & & & & & & & & 0 & 7,0 & 60,000 & \\
\hline 20 & 14 & Estudiante 14 & & 0,0 & & & & & & & & & & & 0 & 7,0 & $60,0 \%$ & \\
\hline 21 & 15 & Estudiante 15 & & 0,0 & & & & & & & & & & & 0 & 7,0 & 60,035 & \\
\hline 22 & & & & & & & & & & & & & & & 0 & 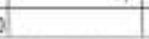 & & . \\
\hline . & - & - E Eastoru & Esadiver. & & uffolet & Moids & Futay & nemies & Corpazaso & ads acezas & Dahenti & & carpar & weod & Nuras cons & 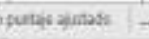 & $\rightarrow$ (4) & \\
\hline
\end{tabular}

Tabla. 7: Parte derecha de la hoja "Pautas y puntajes.

En la columna " $\mathrm{N}^{\mathrm{o}}$ datos", se calculan los datos numéricos ingresados por cada estudiante. Al lado derecho de la casilla "Max individual", se calcula el puntaje máximo del grupo curso, dato que es utilizado para calcular diferentes escalas de notas, y también para mostrar otro dato de interés en una retroalimentación general.

En la columna "Nota Max", se muestra la nota máxima de la evaluación, la que es copiada por defecto desde la hoja "Comparación de escalas" que se explica más adelante". En esta hoja (Pautas y puntajes), dicho parámetro puede modificarse manualmente por cada estudiante en esta hoja, si es necesario.

En la columna “\% exigencia”, se muestra el porcentaje de exigencia de la evaluación, que es copiado por defecto desde la hoja "Comparación de escalas" que se explica más adelante". En esta hoja (Pautas y puntajes), dicho parámetro puede modificarse manualmente por cada estudiante en esta hoja, si es necesario.

Nótese que hay muchas columnas que se han ocultado para facilitar la visualización de la hoja mostrada. 


\begin{tabular}{|c|c|c|c|c|c|c|c|c|c|c|c|c|c|c|c|c|c|}
\hline 4 & A & B & & C & D & E & $F$ & G & H & 1 & 1 & k. & $\mathrm{t}$ & $M$ & $\mathrm{AH}$ & (A) & A) \\
\hline 1 & & Aprobacion. & & & $16.2 \%$ & $04,1 \%$ & $81, T \%$ & $n 7,0 \%$ & $79,9 \%$ & $72,3 \%$ & $50,5 \%$ & $62.3 \%$ & $86,7 \%$ & $53,3 \%$ & & & \\
\hline 2 & & $N^{2}$ pregunts & & & 1 & 2 & 3 & 4 & 5 & 6 & 7 & I & 9 & 10 & & Max prin fotal & 17,25 \\
\hline 3 & & Tipo & & & 1 & 1 & c & c & d & d. & $t$ & 1 & c. & e & & Maxpan ajs. & 16,6 \\
\hline 4 & & Habibidad & & & 1 & 1 & 2 & 2 & 3 & 3 & 4 & 4 & 5 & 6 & $N^{*}$ datos & Max inelividuas & 165 \\
\hline 5 & & Puntaje total & & 18 & 2 & 2 & 3 & 1 & 1 & 1 & 2 & 2 & 2 & 2 & 10 & \\
\hline 6 & 1. & Puntaje aisatado & 7 & $16,2$. & 27 & 20 & $3=$ & 14 & 10 & 10 & 1,78 & 1,768 & $1,85=$ & 1,4 * & 17 & \multicolumn{2}{|c|}{ - Sexigene. } \\
\hline 3 & 1 & Estudiante 1 & & 12,7 & 16 & 1,2 & 1,9 & 09. & 10 & 00 & 1,4 & 15 & 19 & 1,4 & 10 & 70 & $600 \%$ \\
\hline$B$ & 2 & Estudiante 2 & & 16,5 & 20 & 2,9 & 30 & 10 & 10 & 10 & 1,7 & 18. & 1.8 & 1.3 & 10 & 70 & 60,056 \\
\hline 9 & 3. & Estudiante 3 & & 16,1 & 1.5 & 15 & 2.3 & 09 & 19 & 0,8 & 1.4 & 15 & 19 & 1,4 & 10. & 7.0 & 60.05 \\
\hline 10 & 4 & Estudiante 4 & & 14,7 & 17 & 20 & 2,8 & 09. & 0,7 & 0.9 & 15 & 1.5 & 1,5 & 1,3 & 10. & 7,0 & $60.0 \%$ \\
\hline 11 & 5 & Estudiante 5 & & 14,5 & 19 & 19 & 2,4 & 10 & 0.18 & 0.9 & 1,4 & 15 & 14 & 1,7 & 10 & 7,0 & $600 \%$ \\
\hline 12 & 6 & Eitudiante 6 & & 14,3 & 19 & 1,9 & 24 & 10 & $0 B$ & 09 & 1.4 & 15 & 1,4 & 1,1 & 10 & 70 & $60.0 \%$ \\
\hline 13 & 7 & Estudiante 7 & & 5,9 & 0.8 & 0:日 & 0.9 & 06 & 0,3 & ge & 0.5 & Q.7 & 0,0 & 0.5 & 10 & 70 & 60,056 \\
\hline 14 & 8 & Estudiante 8 & & 14.3 & 20. & 1.9 & 2.8 & 00 & 0,9 & 0,8 & 1.2 & 13 & 1,3 & 1.3 & 10. & 7,0 & 60000 \\
\hline 15 & 9 & Estudiante 9 & & 14,3 & 20 & 4,9 & 30 & 09 & 0.9 & 0.9 & 1,2 & 12 & 1,3 & 1,3 & 10 & 7,0 & $60.0 \%$ \\
\hline 16 & 10 & Estudiante 10 & & 145 & 1,9 & 1.9 & 30 & 0.9 & 0.9 & 0.9 & 13 & 13 & 1,3 & 1,2 & 10 & 7,0 & $600 \%$ \\
\hline 17 & 11 & Estudiante 11 & & 14.2 & 20 & 1,8 & 30 & 10 & 0.9 & 10 & 12 & 1,1 & 1,2 & 1,1 & 10 & 70 & $600 \%$ \\
\hline 18 & 12. & Estudiante 12 & & 5,0 & 0,7 & 0,7 & 0.8 & 06 & 0,3 & 0,3 & 0.5 & 0.5. & 0.7 & 180 & 10 & 70 & 60.056 \\
\hline 19 & 13 & Estudiante 13 & & 13,9 & 20 & 1.9 & 2.8 & 10 & 0.8 & 0,8 & 13 & 12 & 12 & 1,1 & 10 & 70 & $6000 \%$ \\
\hline 20 & 11 & Estudiante 14 & & 13,9 & 20. & tie & 30 & 0.8. & 0.9 & 0,8 & 1,1 & 13 & 1,2 & 10 & 10 & 7,0 & $60.0 \mathrm{~s}$ \\
\hline 21 & 15 & Estudiante 15 & & 13,2 & 1,2 & 20 & 2.6 & 0.9 & $0 . \pi$ & 0,8 & 13 & 1,1 & 1,0 & 0,9 & 10 & 7,0 & $600 \%$ \\
\hline 22 & & & & & & & & & & & & & & & o) & & \\
\hline , & , & -. tantere ? & & & numb: & Findes: & $\operatorname{tm}$ & awe $\mathrm{r}$ & fituan: : & fitan & & & inta y purtaj & & ades & Deten - & \\
\hline
\end{tabular}

Tabla.8: Hoja "Pautas y puntajes" con los puntajes ingresados por estudiantes. Los puntajes obtenidos de las revisiones efectuadas según lo indicado en Fig-4, se copian y pegan en esta sección (usando la función de pegar valores y formatos). En este caso, para el ejemplo, se han puesto puntajes ficticios para destacar ciertos aspectos del HAPRE. Según sean los puntajes obtenidos, las casillas respectivas se colorean automáticamente. Mientras más cerca esté el puntaje obtenido por el estudiante, respecto al puntaje de la pauta, la casilla será más verde; por ende, mientras más alejado, será más rojo. De la misma forma, en la fila de "Aprobación", los porcentajes de aprobación se calculan automáticamente y las respectivas casillas se colorean de la misma forma que las descritas para los puntajes de los estudiantes.

Ahora aparece un valor en la casilla a la derecha de la que dice "Puntaje ajustado". Este puntaje corresponde a la suma total de todos los máximos puntajes obtenidos en cada pregunta, por el grupo-curso. A la derecha, cerca de la esquina superior, aparecen valores para otros parámetros:

- Max prm total: Puntaje promedio entre Puntaje total y Max individual.

- Max prm ajs: Puntaje promedio entre Puntaje ajustado y Max individual.

- Max individual: Puntaje máximo de un estudiante del grupo-curso.

Estos tres parámetros serán utilizados para calcular diferentes escalas de notas que HAPRE ofrece para el docente. 
Determinando las calificaciones

En la siguiente hoja del HAPRE, llamada "Comparación de escalas", se muestran gráficos y tablas para que el docente pueda determinar la más adecuada escala de notas para el grupo-curso.

\begin{tabular}{|c|c|c|c|c|c|c|c|c|}
\hline$\Delta$ & $\mathrm{A}$ & B & $\mathrm{C}$ & D & E & G & $\mathrm{H}$ & I \\
\hline 1 & & Puntaje de referencia & & 18,0 & 17,3 & 16,7 & 16,6 & 16,5 \\
\hline 2 & & Puntaje de aprobación & & 10,8 & 10,4 & 10,0 & 10,0 & 9,9 \\
\hline 3 & & Promedio & 13,1 & 5,1 & 5,3 & 5,5 & 5,6 & 5,6 \\
\hline 4 & $\overrightarrow{n+1}$ & Estudiante & Puntaj & E1 & E2 & E3 & E4 & E5 \\
\hline 5 & 1 & Estudiante 1 & 12,7 & 4,8 & 5,0 & 5,2 & 5,2 & 5,3 \\
\hline 6 & 2 & Estudiante 2 & 16,5 & 6,4 & 6,7 & 6,9 & 7,0 & 7,0 \\
\hline 7 & 3 & Estudiante 3 & 14,1 & 5,4 & 5,6 & 5,8 & 5,9 & 5,9 \\
\hline 8 & 4 & Estudiante 4 & 14,7 & 5,6 & 5,9 & 6,1 & 6,1 & 6,2 \\
\hline 9 & 5 & Estudiante 5 & 14,5 & 5,5 & 5,8 & 6,0 & 6,1 & 6,1 \\
\hline 10 & 6 & Estudiante 6 & 14,3 & 5,5 & 5,7 & 5,9 & 6,0 & 6,0 \\
\hline 11 & 7 & Estudiante 7 & 5,9 & 3,1 & 3,1 & 3,2 & 3,2 & 3,2 \\
\hline 12 & 8 & Estudiante 8 & 14,3 & 5,5 & 5,7 & 5,9 & 6,0 & 6,0 \\
\hline 13 & 9 & Estudiante 9 & 14,3 & 5,5 & 5,7 & 5,9 & 6,0 & 6,0 \\
\hline 14 & 10 & Estudiante 10 & 14,5 & 5,5 & 5,8 & 6,0 & 6,1 & 6,1 \\
\hline 15 & 11 & Estudiante 11 & 14,2 & 5,4 & 5,7 & 5,9 & 5,9 & 5,9 \\
\hline 16 & 12 & Estudiante 12 & 5,0 & 2,9 & 3,0 & 3,0 & 3,0 & 3,0 \\
\hline 17 & 13 & Estudiante 13 & 13,9 & 5,3 & 5,5 & 5,7 & 5,8 & 5,8 \\
\hline 18 & 14 & Estudiante 14 & 13,9 & 5,3 & 5,5 & 5,7 & 5,8 & 5,8 \\
\hline 19 & 15 & Estudiante 15 & 13,2 & 5,0 & 5,2 & 5,4 & 5,4 & 5,5 \\
\hline 20 & & & & & & & & \\
\hline
\end{tabular}

Tabla.9: Parte izquierda de la hoja "Comparación de escalas", en la que HAPRE muestra diferentes escalas de notas en forma simultánea. Todas las escalas consideran los siguientes parámetros:

Puntaje de referencia, señalado en la parte superior de la tabla, que indica el puntaje equivalente a la nota máxima de la evaluación, la cual está seteada en otra sección de esta hoja; en este caso, es 7,0 por defecto.

Puntaje de aprobación, señalado en la parte superior de la tabla (debajo de Puntaje de referencia), que indica el puntaje equivalente a la nota mínima de aprobación. Esta tiene directa relación con el porcentaje de exigencia; que está seteada en otra sección de esta hoja; en este caso, es $60 \%$ por defecto. HAPRE calcula $60 \%$ del Puntaje de referencia de la evaluación, y a ese puntaje se le asigna la nota de aprobación que está seteada en otra sección de esta hoja; en este caso, es 4,0 por defecto.

Nota mínima, que esta seteada en otra sección de esta hoja, junto con los parámetros anteriores; en este caso, es 2,0 por defecto. 
Considerando los parámetros anteriores, las 5 escalas presentadas se diferencian principalmente en el Puntaje de referencia, según la siguiente tabla:

\begin{tabular}{|l|l|}
\hline Escala & Puntaje equivalente a la nota máxima. \\
\hline E1 & Máximo puntaje de la evaluación. \\
\hline E2 & $\begin{array}{l}\text { Promedio entre puntaje máximo de la evaluación y puntaje máximo de un } \\
\text { estudiante en el grupo curso. }\end{array}$ \\
\hline E3 & Puntaje ajustado de la evaluación. \\
\hline E4 & $\begin{array}{l}\text { Promedio entre puntaje ajustado de la evaluación y puntaje máximo de un } \\
\text { estudiante en el grupo curso. }\end{array}$ \\
\hline E5 & Puntaje máximo de un estudiante en el grupo curso. \\
\hline
\end{tabular}

Gracias a la función de filtro en Excel, la tabla puede ser ordenada para mostrar los resultados de mayor a menor, o viceversa.

\begin{tabular}{|c|c|c|c|c|c|c|c|c|c|}
\hline$\Delta$ & A & B & c & D & $\mathrm{E}$ & G & $\mathrm{H}$ & 1 & J \\
\hline 1 & & Puntaje de referencia & & 18,0 & 17,3 & 16,7 & 16,6 & 16,5 & \\
\hline 2 & & Puntaje de aprobación & & 10,8 & 10,4 & 10,0 & 10,0 & 9,9 & \\
\hline 3 & & Promedio & 13,1 & 5,1 & 5,3 & 5,5 & 5,6 & 5,6 & \\
\hline 4 & $\overrightarrow{7}$ & Estudiante & Puntaj & E1 & E2 V & E3 1 & E4 & E5 & \\
\hline 5 & 2 & Estudiante 2 & 16,5 & 6,4 & 6,7 & 6,9 & 7,0 & 7,0 & \\
\hline 6 & 4 & Estudiante 4 & 14,7 & 5,6 & 5,9 & 6,1 & 6,1 & 6,2 & \\
\hline 7 & 10 & Estudiante 10 & 14,5 & 5,5 & 5,8 & 6,0 & 6,1 & 6,1 & \\
\hline 8 & 5 & Estudiante 5 & 14,5 & 5,5 & 5,8 & 6,0 & 6,1 & 6,1 & \\
\hline 9 & 9 & Estudiante 9 & 14,3 & 5,5 & 5,7 & 5,9 & 6,0 & 6,0 & \\
\hline 10 & 6 & Estudiante 6 & 14,3 & 5,5 & 5,7 & 5,9 & 6,0 & 6,0 & \\
\hline 11 & 8 & Estudiante 8 & 14,3 & 5,5 & 5,7 & 5,9 & 6,0 & 6,0 & \\
\hline 12 & 11 & Estudiante 11 & 14,2 & 5,4 & 5,7 & 5,9 & 5,9 & 5,9 & \\
\hline 13 & 3 & Estudiante 3 & 14,1 & 5,4 & 5,6 & 5,8 & 5,9 & 5,9 & \\
\hline 14 & 13 & Estudiante 13 & 13,9 & 5,3 & 5,5 & 5,7 & 5,8 & 5,8 & \\
\hline 15 & 14 & Estudiante 14 & 13,9 & 5,3 & 5,5 & 5,7 & 5,8 & 5,8 & \\
\hline 16 & 15 & Estudiante 15 & 13,2 & 5,0 & 5,2 & 5,4 & 5,4 & 5,5 & \\
\hline 17 & 1 & Estudiante 1 & 12,7 & 4,8 & 5,0 & 5,2 & 5,2 & 5,3 & \\
\hline 18 & 7 & Estudiante 7 & 5,9 & 3,1 & 3,1 & 3,2 & 3,2 & 3,2 & \\
\hline 19 & 12 & Estudiante 12 & 5,0 & 2,9 & 3,0 & 3,0 & 3,0 & 3,0 & \\
\hline 55 & & & & & & & & & \\
\hline
\end{tabular}

Tabla.10: Sección izquierda de la hoja "Comparación de escalas". Ahora se muestra con 2 filtros aplicados; se han eliminado las celdas vacías y la lista de estudiantes se muestra en orden decreciente según el puntaje obtenido en la evaluación, lo que permite visualizar mejor las calificaciones en las diferentes escalas. 


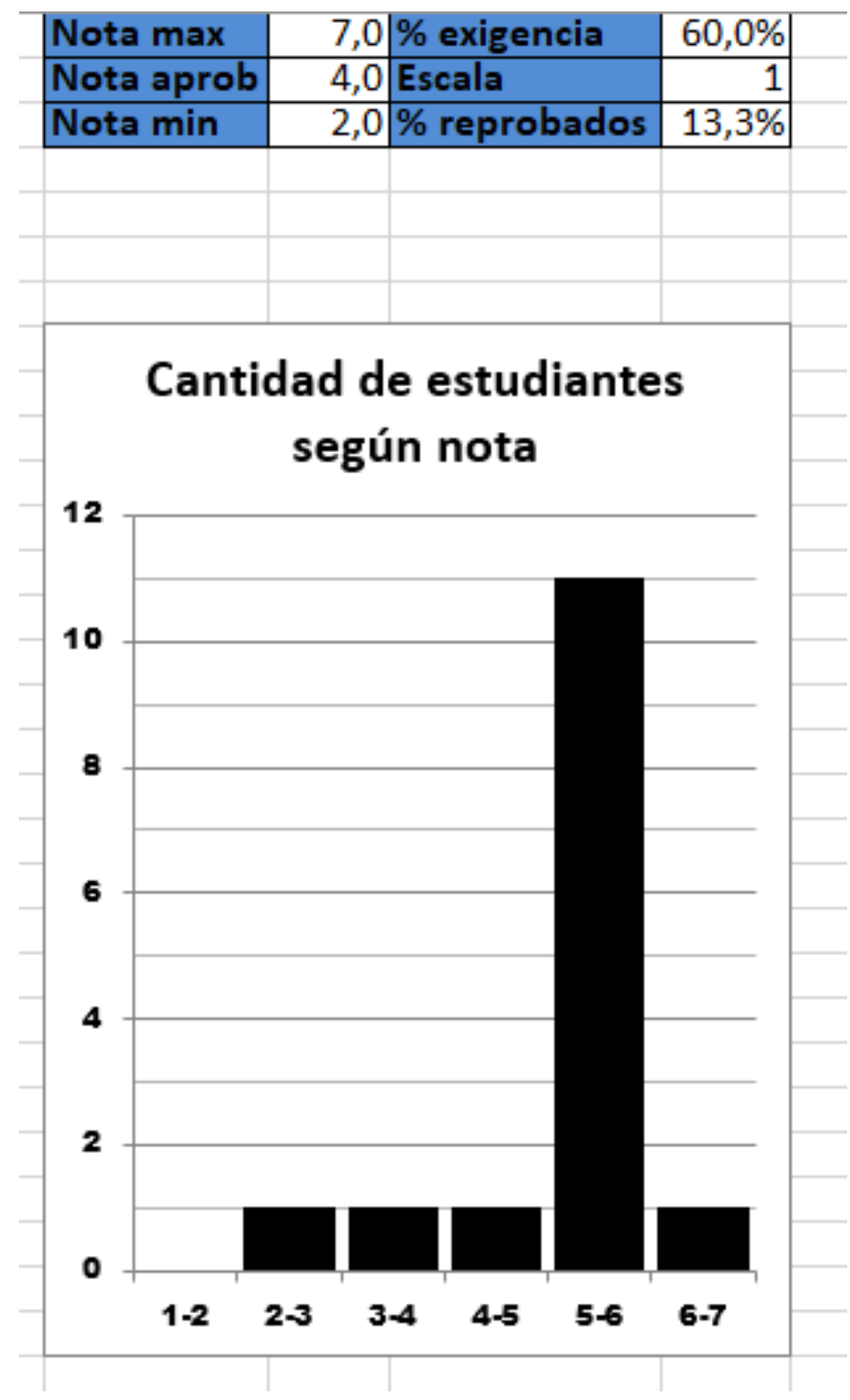

Gráfico.1: Sección central de la hoja "Comparación de escalas". En la tabla de arriba se indican diferentes parámetros esenciales para determinar las calificaciones; si bien están presentados por defecto, pueden ser modificados si es necesario. Excepto el indicador “\% reprobados" que es calculado en forma autónoma por HAPRE. Este parámetro indica la cantidad de estudiantes del grupo curso que obtuvieron una calificación menor a la nota de aprobación. Dicho porcentaje puede variar si se alteran los parámetros anteriores. En "Escala", el docente debe escoger si mantiene la escala 1 , o selecciona otra alternativa.

Abajo, el gráfico de barras indica la distribución de estudiantes en el rango de calificaciones indicado en el eje X. Dicha distribución variará si se cambian los parámetros de evaluación presentados en la tabla anterior. 
Propuesta de una herramienta informática...

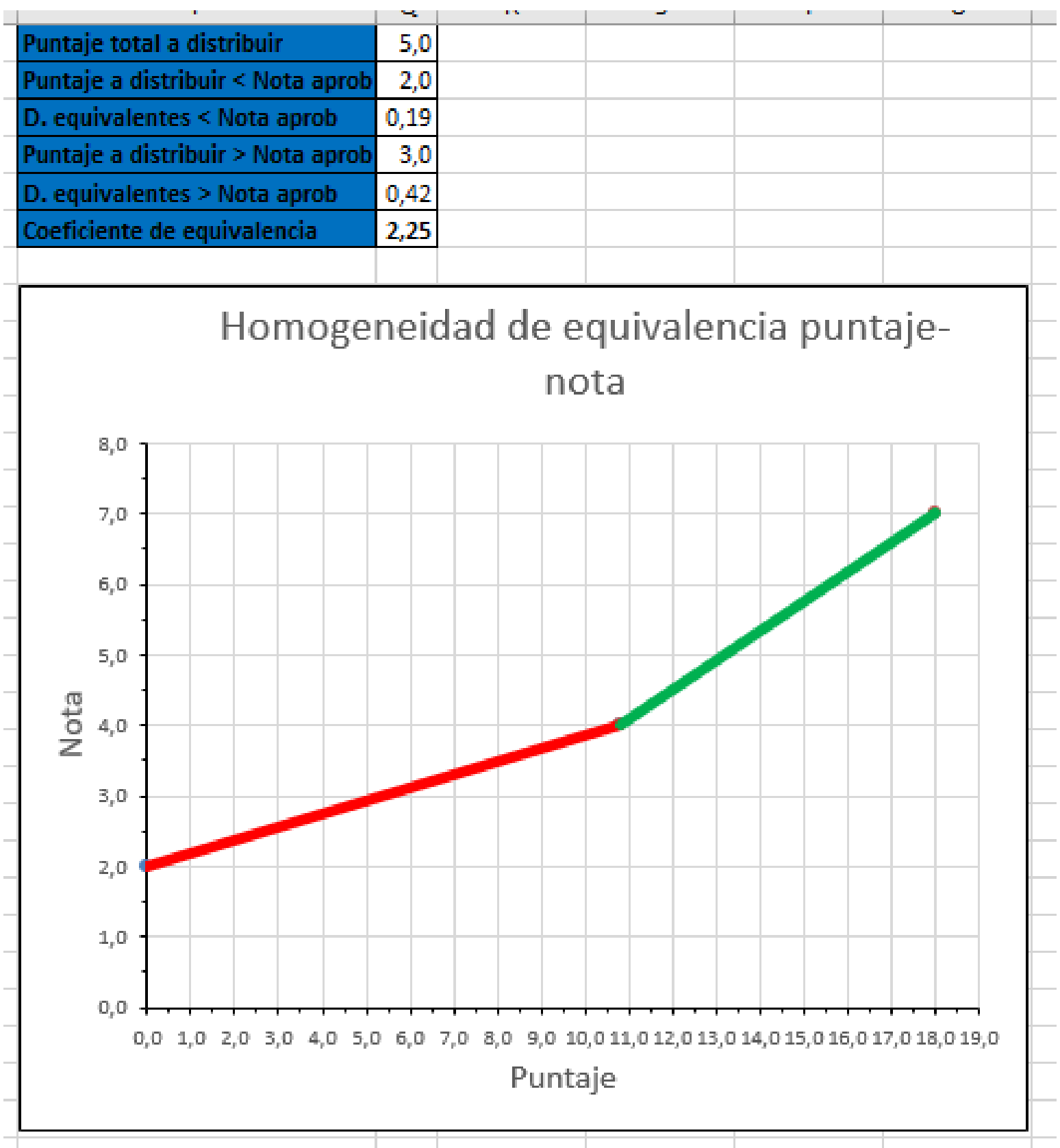

Gráfico.2: Sección derecha de la hoja "Comparación de escalas", en la que ve el efecto del porcentaje de exigencia, en las calificaciones. La tabla arriba del gráfico se genera a partir de los parámetros de la tabla descrita en el gráfico 1.

Puntaje total para distribuir: Corresponde a la resta entre la Nota max de la evaluación con la Nota min. El resultado es la fracción de la nota que está en juego en la evaluación. Puntaje para distribuir < Nota aprob: Corresponde a la resta entre la Nota aprob de la evaluación y la Nota min. El resultado es la fracción de la nota que está en juego en la evaluación, para conseguir la nota de aprobación previamente seteada. 
D. equivalentes < Nota aprob: Corresponde a un coeficiente entre el parámetro anterior y el puntaje de aprobación. Esencialmente, el resultado indica a cuantas décimas de la calificación equivale 1 punto de la evaluación que sea menor al puntaje de aprobación de esta. En este caso, en la revisión de esta evaluación, cada punto ganado desde 0 hasta los 10,8 (puntaje de aprobación según la escala seteada) aporta 0,19 décimas de calificación. Puntaje para distribuir > Nota aprob: Corresponde a la resta entre la Nota max de la evaluación y la Nota aprob. El resultado es la fracción de la nota que está en juego en la evaluación, para conseguir la nota máxima previamente seteada, una vez alcanzada la nota de aprobación.

D. equivalentes > Nota aprob: Corresponde a un coeficiente entre el parámetro anterior, y la resta entre el puntaje máximo de la evaluación con el puntaje de aprobación. Esencialmente, el resultado indica a cuantas décimas de la calificación equivale 1 punto de la evaluación que sea mayor al puntaje de aprobación de esta. En este caso, en la revisión de esta evaluación, cada punto ganado desde los 10,8 (puntaje de aprobación según la escala seteada) hasta los 18 (puntaje máximo según la escala seteada) aporta 0,42 décimas de calificación.

Coeficiente de equivalencia: Corresponde a la división entre "D. equivalentes $>$ Nota aprob" y "D. equivalentes < Nota aprob". Este parámetro indica la equivalencia en décimas de nota, de un punto de la evaluación obtenido sobre el puntaje de aprobación, comparado con un punto de la evaluación obtenido bajo el puntaje de aprobación. En este caso, dicho coeficiente es de 2,25. Con los parámetros seteados en la tabla de la Fig-11, significa que si 1 punto hasta los 10,8 equivalieran a 1 décima de nota; 1 punto sobre los 10,8 equivaldrían a 2,25 décimas de nota. Si se quisiera que cada punto de la evaluación equivaliera a la misma cantidad de décimas de la calificación, sin importar si estuviera bajo o sobre el puntaje de aprobación, dicho coeficiente debería tener un valor de 1 o muy cercano.

Abajo se muestra el gráfico que muestra como asciende la calificación en función del puntaje. La pendiente roja muestra ese ascenso hasta el puntaje de aprobación; la pendiente verde muestra ese ascenso desde el puntaje de aprobación hasta el puntaje máximo. Con los parámetros seteados, el gráfico indica que la calificación asciende más lentamente al principio, hasta alcanzar el puntaje de aprobación; luego, la pendiente es mayor, indicando un ascenso más rápido. 
Esta sección muestra al docente si el porcentaje de exigencia se ajusta al criterio que quiere aplicar para asignar las calificaciones.

Calificaciones oficiales

\begin{tabular}{|c|c|c|c|c|c|c|}
\hline$\Delta$ & A & $B$ & $\mathrm{C}$ & $\mathrm{D}$ & $\mathrm{E}$ & $\mathrm{F}$ \\
\hline 1 & & Estudiante & Puntaje & Nota & Extra & Definitivas \\
\hline 2 & 1 & Estudiante 1 & 12,7 & 4,8 & & 4,8 \\
\hline 3 & 2 & Estudiante 2 & 16,5 & 6,4 & 0,6 & 7,0 \\
\hline 4 & 3 & \begin{tabular}{|l|} 
Estudiante 3 \\
\end{tabular} & 14,1 & 5,4 & & 5,4 \\
\hline 5 & 4 & Estudiante 4 & 14,7 & 5,6 & & 5,6 \\
\hline 6 & 5 & Estudiante 5 & 14,5 & 5,5 & & 5,5 \\
\hline 7 & 6 & Estudiante 6 & 14,3 & 5,5 & & 5,5 \\
\hline 8 & 7 & Estudiante 7 & 5,9 & 3,1 & 1 & 4,1 \\
\hline 9 & 8 & Estudiante 8 & 14,3 & 5,5 & & 5,5 \\
\hline 10 & 9 & Estudiante 9 & 14,3 & 5,5 & & 5,5 \\
\hline 11 & 10 & Estudiante 10 & 14,5 & 5,5 & 1 & 6,5 \\
\hline 12 & 11 & Estudiante 11 & 14,2 & 5,4 & & 5,4 \\
\hline 13 & 12 & Estudiante 12 & 5,0 & 2,9 & & 2,9 \\
\hline 14 & 13 & Estudiante 13 & 13,9 & 5,3 & & 5,3 \\
\hline 15 & 14 & Estudiante 14 & 13,9 & 5,3 & & 5,3 \\
\hline 16 & 15 & Estudiante 15 & 13,2 & 5,0 & 0,6 & 5,6 \\
\hline \multicolumn{7}{|l|}{17} \\
\hline 18 & & & & & & \\
\hline 10 & & & & & & \\
\hline
\end{tabular}

Tabla.11: Sección izquierda de la hoja "Definitivas". Aquí se muestra misma lista de todos los estudiantes que la ingresada en la hoja "Pautas y puntajes". En la columna "Puntaje", sus puntajes respectivos. En la columna "Nota" se muestran las calificaciones resultantes según los parámetros establecidos en la hoja anterior ("Comparación de escalas"). La columna "Extra" permite incorporar puntajes adicionales obtenidos como premios, o incluso una nota adicional a ponderar con la nota de la presente evaluación. La columna "Definitivas" muestra las calificaciones con todas las consideraciones efectuadas; es decir, las notas que se informan oficialmente. 


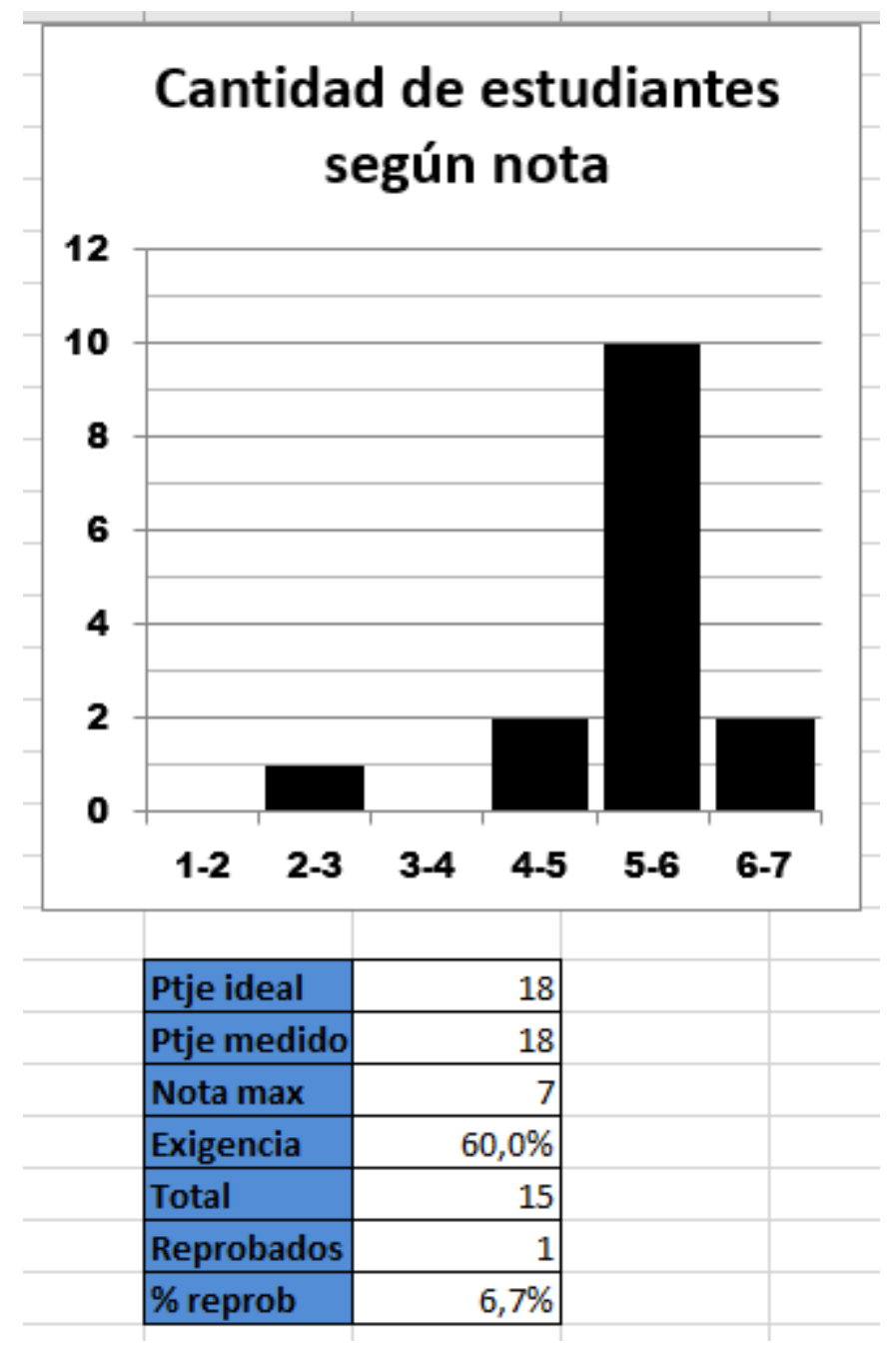

Gráfico.3: Sección central de la hoja "Definitivas". En la tabla de abajo se indica un resumen con los parámetros medidos y resultados obtenidos, uno de interés es el porcentaje de reprobados, el cual está determinado según las notas de la columna "Definitivas". En esas bases resulta el gráfico de barras arriba, que muestra la distribución de estudiantes en el rango de calificaciones indicado en el eje $\mathrm{X}$. Dicha distribución variará si se cambian los parámetros descritos anteriormente. Nótese que, debido a los puntajes considerados, el gráfico de distribución en esta hoja es diferente del obtenido en la hoja "Comparación de escalas", el que no considera la inclusión de algún extra en la calificación. 


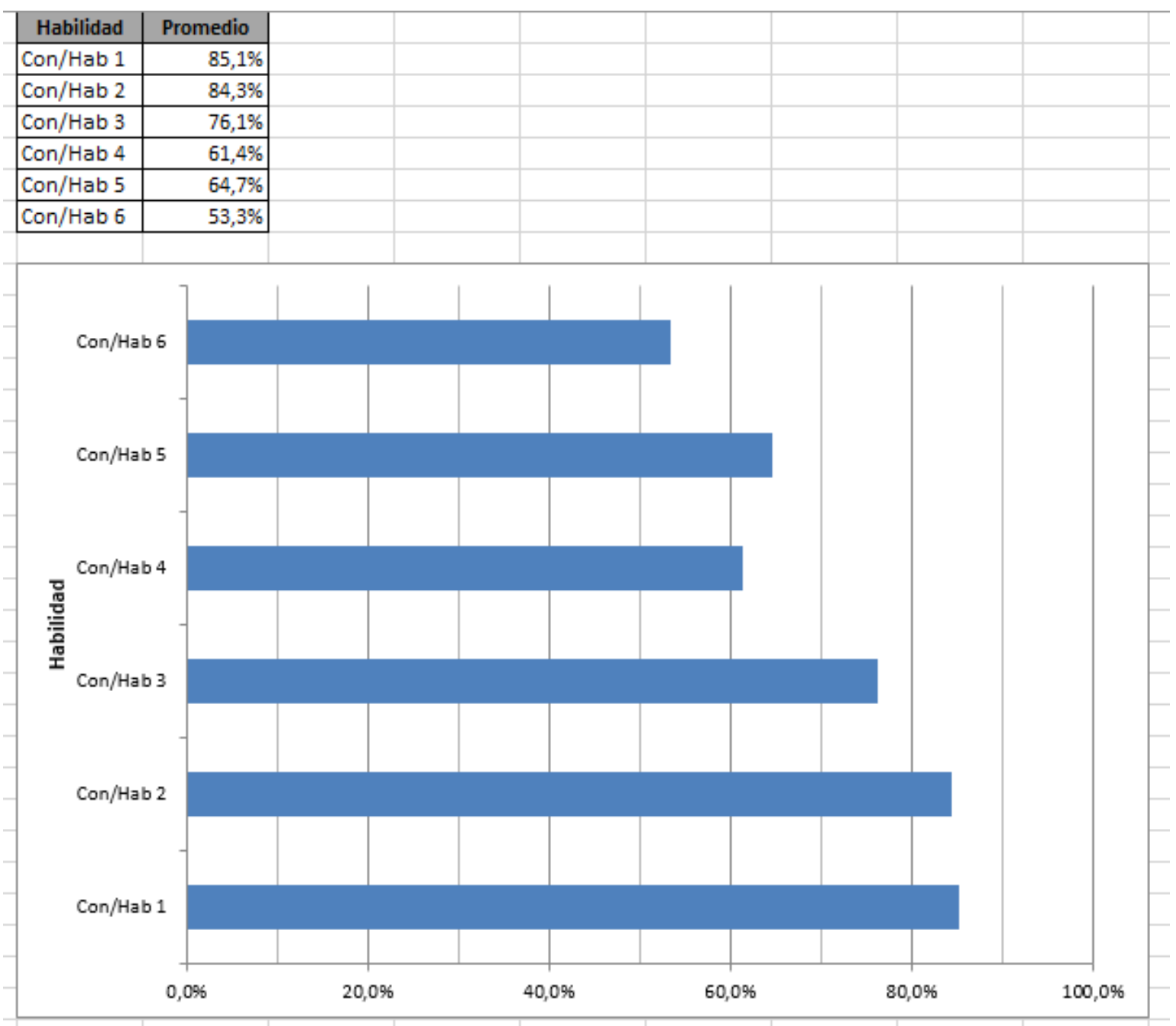

Grafico.4: Sección derecha de la hoja "Definitivas". La tabla arriba muestra los porcentajes de aprobación del grupo-curso, según el Con/Hab especificado por pregunta, en la hoja "Pautas y puntajes". El gráfico abajo permite visualizar comparativamente mejor entre la aprobación de un Con/Hab y otro.

\section{DISCUSIÓN}

HAPRE es una herramienta informática en forma de un libro de cálculo; que funciona en forma similar a otros softwares que ayudan a la revisión y retroalimentación de evaluaciones; pero este software ¿permite también la revisión y retroalimentación de preguntas de selección múltiple? De hecho, así es. No se ha encontrado en alguna investigación previa, una propuesta similar a la planteada aquí. Por lo tanto, esta herramienta se ha elaborado bajo los parámetros de su propuesta madre, sin encontrar similitudes desde el punto de los objetivos y de su fin. Existen herramientas que mejoran la gestión pedagógica desde el punto de vista de los instrumentos de evaluaciones de ítems 
de alternativas o de respuestas abierta (también conocida como evaluación de desarrollo). En otras palabras, HAPRE permite la revisión y retroalimentación de todo tipo de evaluaciones.

HAPRE utiliza el software Microsoft Excel como herramienta operativa y ágil en un contexto de respuestas a las evaluaciones de respuestas abiertas, siendo una gran ventaja que es ampliamente utilizado y de fácil acceso, por lo que se facilita su uso. Aunque tal como se plantea en Delgado, 2009 (17), es necesario que tanto docentes y estudiantes asimilen el uso de este tipo de TIC y puedan manejarlas eficientemente y estén dispuestos a aprender el uso de nuevas herramientas.

La rúbrica es una pieza fundamental, cuyo fin es describir detalladamente los niveles de ejecución esperados, a partir de lo cual se emiten juicios sobre la calidad de la tarea o el nivel de pericia mostrado (Tardif, 2006; Etkina y cols., 2004). No obstante, su potencial, Reddy y Andrade (2010) subrayan la falta de rigor en la elaboración de las rúbricas que repercutan en el uso de estos instrumentos para evaluar. Por tanto, se deduce la utilidad de una rúbrica para distinguir niveles de desempeño de la competencia argumentativa en respuestas abiertas para verificar los niveles de logros alcanzados por los estudiantes frente a este tipo de preguntas. La rúbrica sugerida en esta propuesta destaca ser la más apropiada para ser utilizada en estos parámetros y en los análisis de cada respuesta.

El ingreso de los puntajes puede ser ajustado según la naturaleza de la evaluación. La hoja "Molde" presentada en esta propuesta, está pensada para ingresar dichos puntajes desde una evaluación de respuesta abierta. Sin embargo, los algoritmos pueden ser reescritos para ingresar puntajes de otro tipo de evaluación de respuesta cerrada, como los de una evaluación de selección múltiple. En otras palabras, HAPRE puede ser adaptado a cualquier situación requerida.

HAPRE, permite al docente especificaciones de revisión de la evaluación, como los aspectos a evaluar de cada pregunta y su ponderación correspondiente, cuando se consigna la ponderación hay que tener en consideración variables externas e internas. Sin embargo, La ponderación puede cambiar la estructura de los datos si incluimos a los y a las estudiantes con necesidades educativas especiales (NEE), por lo que se debe tener precaución al ser aplicada y dependerá de que porcentaje de logro tengamos que insertar, para optimizar HAPRE y por consiguiente también diferenciar un contexto de inclusión. 
Además, HAPRE permite hacer este ajuste en forma individual, haciendo ajustes como en el porcentaje de exigencia de un solo estudiante en particular.

El docente se dedica a ingresar las ponderaciones y los niveles de logro por pregunta. Mientras HAPRE hace el resto de los cálculos para determinar los puntajes respectivos. ¿Pero es confiable dejarle ese trabajo a una hoja de cálculo de Excel? Definitivamente sí. Se debe dejar establecido a los estudiantes que el cálculo que efectúa Excel es completamente seguro, aunque existe la probabilidad de que haya un error humano, por ejemplo, al valorar un nivel de logro. Dicho lo anterior, siempre será importante y necesario transparentar la revisión que haya hecho el docente, para que el estudiante pueda ejercer su derecho a desacuerdo, si lo estima conveniente.

En la hoja "Pauta y puntajes" de HAPRE, es donde se ingresan los puntajes respectivos para un análisis del grupo-curso en general. Cuando se formula que HAPRE puede darnos un muestreo gráfico para un análisis de grupo de curso, estamos frente a un procedimiento muy importante dentro de las conjeturas de la utilización de una herramienta como Excel, para otorgar un fenómeno que solo se podría visualizar con un análisis de un docente.

En esta instancia, HAPRE entrega una retroalimentación visualmente fácil de entender, ya sea para el grupo-curso en general, o para un solo estudiante en particular.

Se consigna que esta forma de trabajo está pensada para facilitar el actuar docente, evitando que este tenga que hacer la operatoria matemática de los puntajes, a la vez que esta hoja sirve de retroalimentación para los futuros resultados.

Cuando los docentes tienen que calificar, se viene un cuestionamiento si es justo o injusto la calificación. Dicho lo anterior, cada docente tiene que calificar objetivamente siendo esta una escala establecida, expresado mediante una denominación o una puntuación, que se asigna a una persona para valorar el nivel de suficiencia o insuficiencia de los conocimientos o formación mostrados en un examen, un ejercicio o una prueba. HAPRE cuenta con un parámetro que permite una evaluación más justa a la realidad del grupocurso, llamado "Puntaje ajustado" ¿Qué significa en la práctica? corresponde a la suma total de todos los máximos puntajes obtenidos en cada pregunta, por el grupo-curso, esto permite que el docente puede establecer una escala máxima de puntaje que se ajuste al nivel que el grupo-curso puede alcanzar en la realidad, y por tanto, ajustando las calificaciones obtenidas dentro de ese grupo-curso en particular. 
El docente formula una escala que consiste en una serie de indicadores y una escala graduada para evaluar a cada uno de sus estudiantes. La escala de calificación puede ser numérica, literal, gráfica y descriptiva. HAPRE trabaja con escalas formuladas por el docente, por tanto, ¿Cómo se determina una escala? Considerando los parámetros anteriores, las 5 escalas presentadas se diferencian principalmente en el Puntaje de referencia. Las escalas están ordenadas desde la más exigente a la menos exigente. HAPRE muestra todas las escalas en paralelo para que el docente las compare todas a la vez, de esa forma puede determinar cuál es la mejor escala que se ajuste a la realidad del grupo-curso, y de esa forma, establecer las calificaciones más justas, aunque esto último dependerá solo del criterio establecido por el docente mismo o el que se apegue a la institución educacional. Una decisión que será mejor pensada con todos los datos a la vista y comparados al mismo tiempo.

El docente busca la homogeneidad de las equivalencias de las calificaciones, por tanto, nace un cuestionamiento si realmente el $60 \%$ es válido para una justa calificación. Entonces, ¿Cuál es el sentido del 60\%? Que el estudiante apruebe una evaluación demostrando que domina más del 50\% de los contenidos en una determinada materia. No existe una norma establecida que exija a todos los docentes un determinado porcentaje de exigencia en las evaluaciones; esto siempre depende del criterio del docente, aunque se ha visto una tendencia a que este sea $50 \%$ o más. Uno de los parámetros de evaluación que HAPRE permite ajustar es el porcentaje de exigencia. Para que el docente nivele este porcentaje lo más justo posible, HAPRE cuenta con una herramienta que permite ver la equidad en la distribución de las décimas de la calificación correspondiente.

Una de las mayores fortalezas de HAPRE es la capacidad de ordenar y presentar los resultados para facilitar la retroalimentación adecuada tanto para estudiantes como para el docente. Tal como se muestra en el estudio de Espinoza, 2021 (34), la retroalimentación es un factor relevante en el proceso de enseñanza-aprendizaje, pero generalmente queda en informar una calificación, mostrando solo una parte del resultado de la evaluación ((Saiz-Linares \& Susinos-Lara, 2018). Citado en 34).

Considerando el limitado tiempo pedagógico que hay en una clase de nivel escolar, es crucial presentar los resultados de la evaluación en forma completa, pero visualmente entendible por todos. Según lo citado en (35), existen varios tipos de retroalimentación útiles y HAPRE cumple con facilitar varios de los mencionados; así, la retroalimentación 
explícita queda demostrada en diferentes gráficos, datos numéricos en escalas de colores y/o gráficos de barras que rápidamente pueden mostrar los aciertos y equivocaciones del grupo-curso; y también en cada hoja donde se ingresan los puntajes de cada estudiante, se muestran numérica y gráficamente los aciertos y errores individualmente.

De la interpretación de los gráficos y tabla viene también una retroalimentación implícita que presenta una utilidad para futuras evaluaciones cuando un tipo de error aparece repetidamente, lo que está reforzado por el hecho de que al tratarse de una herramienta digital, los registros se pueden guardar por mucho tiempo, se pueden ordenar fácilmente bajo diferentes criterios y así, vincular los datos en una sola hoja de cálculo, para mostrar el progreso ya sea de un grupo-curso, o de un estudiante en particular.

Mostrar los aciertos y errores particulares, es clave para determinar el curso de acción que posibilite las mejoras en los resultados posteriores y HAPRE tiene el potencial de permitir un monitoreo constante en el tiempo.

Conclusiones.

El uso de la inteligencia artificial en la educación plantea paradigmas legítimos sobre cómo se recolectan y usan los datos calificativos. Dicho lo anterior, queda mucho tiempo para el desarrollo de una inteligencia artificial que sea capaz de analizar la redacción y/o desarrollo argumental de una respuesta, y que esta determine por sí sola si dicha respuesta es correcta, de acuerdo con lo esperado del aprendizaje de un cierto contenido. Toda evaluación necesariamente llega a un punto donde la información se reduce al ingreso y obtención de datos y resultados numéricos; en ese aspecto, hemos demostrado a través de la herramienta HAPRE, que un software puede ayudar mucho a un docente en esta etapa del proceso de enseñanza, incluso si se trata de evaluaciones de respuesta abierta.

Aún es necesario que el docente revise las evaluaciones y valore los niveles de logro en cada respuesta, lo que siempre implicará un grado de subjetividad que puede ser discutible por los y las estudiantes. HAPRE permite que los cálculos necesarios para obtener una calificación sean obtenidos casi en tiempo real, además de que, al mismo tiempo, el software está obteniendo toda la información necesaria que el docente pueda necesitar para adaptar las calificaciones a la realidad de cada curso e incluso, a estudiantes con necesidades educativas especiales (NEE) dentro de un curso. En todos los casos, se mejora la eficiencia en esta vital etapa del aprendizaje, además de resultar de un gran apoyo a la labor del docente. 
HAPRE es una herramienta informática hecha para esta propuesta, como una demostración de que es posible disponer de un software que permita acortar la brecha entre la revisión de evaluaciones y la automatización de dicha labor, algo con lo que sí cuentan las evaluaciones de selección múltiple con muchos programas a disposición; y es una de las razones por las que este último tipo de evaluaciones es muy utilizada, permite obtener calificaciones de grandes volúmenes de evaluaciones en corto tiempo; pero estas evaluaciones tienen grandes desventajas como evaluar solo la obtención de una respuesta, sin evaluar el proceso para llegar a dicha respuesta. Como contraparte, las evaluaciones de respuesta abierta sí permiten esta evaluación, al costo de un considerable mayor tiempo. HAPRE permite reducir el tiempo de revisión de preguntas abiertas y a la vez que permite una mejor retroalimentación para los y las estudiantes y para él o la docente. HAPRE es una herramienta informática propuesta, basada exclusivamente en el programa Microsoft Excel, que es un software de amplio uso. Su manejo puede ser aprendido sin dificultades ya que incluso existen tutoriales gratis en la web; por lo que su manejo puede ser incluso más fácil e intuitivo que otros softwares que el mercado pueda disponer, con múltiples formas de personalización a cada docente y/o establecimiento educacional. La propuesta de HAPRE es solo una de tantas formas en que este software puede tener una valiosa aplicación para la labor docente. Puede ser actualizada constantemente además de que, con los algoritmos adecuados, puede incorporar más herramientas estadísticas para mejorar los análisis calificativos y así, una mejor toma de decisiones.

\section{REFERENCIAS BIBLIOGRAFICAS}

Rodríguez Conde, Ma José. (2005) Aplicación de las TIC a la evaluación de alumnos universitarios. Teoría de la Educación. Educación y Cultura en la Sociedad de la Información, vol. 6, núm. 2, Universidad de Salamanca. Salamanca, España

Katherina Edith Gallardo Córdova, María Eugenia Gil Rendón, Baltazar Contreras Durán, Evelyn García Hernández, Roger Alberto Lázaro Hernández y Li-libeth Ocaña Jiménez. (2011). Toma de decisiones para la evaluación formativa: el proceso de planeación y determinación de sus mecanismos. México

Lilliam Perurena Cancio. Mercedes Moráguez Bergues. (2013) Usabilidad de los sitios Web, los métodos y las técnicas para la evaluación. Universidad de La Habana. La Habana, Cuba 
Portal de recursos educativos, tareas, apuntes, monografías, ensayos http://www.webscolar.com

M. Gabriela Vázquez Olivera. (2016). Problemas y limitaciones del uso de pruebas estandarizadas para la evaluación educativa en México

Walter Moreno-Crespo. Nancy Teresa Paredes-Salazar. (2014). La gestión de las TIC y la calidad de la educación, medida por los resultados de las evaluaciones escolares estandarizadas. Colombia

Revista Paraguaya de Educación. (2012). Experiencia de implementación de las tecnologías de la información y comunicación en educación. Paraguay

Jerome Morrissey. (s/a) El uso de TIC en la enseñanza y el aprendizaje. Cuestiones y desafíos.

XX Encuentro internacional Virtual Educa Argentina. (2018). Elaboración de evaluaciones formativas, en el área de las matemáticas mediadas por TIC (softwares estadísticos) con el objetivo de experimentar la práctica profesional. Argentina

Michel Enrique Gamboa Graus. (s/a). Escalas de medición y análisis de datos estadísticos aplicados a la investigación educativa. Cuba

Portal de recursos educativos, tareas, apuntes, monografías, ensayos. http://www.webscolar.com

Sally Heinz. María Inés Lara. (2011). Programa de capacitación en competencias TICs para docentes. Chile

José Gimeno Sacristán. (s/a). ¿Quién Fracasa cuando hay fracaso Escolar?

Bernal García, Juan Jesús. (2011). Representación Automática de Funciones de Excel y su Aplicación Docente. Colombia

Delgado, Mercedes; Arrieta, Xiomara; Riveros, Víctor. (2009). Uso de las TIC en educación, una propuesta para su optimización. Omnia, vol. 15, núm. 3, Universidad del Zulia. Maracaibo, Venezuela

Flor Idalia Lanuza Gámez. (2018). Uso y aplicación de las TIC en el proceso de enseñanza-aprendizaje. Nicaragua

Manuel Fernández Navas. Noelia Alcaraz Salarirche. Miguel Sola Fernández. (2017). Evaluación y Pruebas Estandarizadas: Una Reflexión sobre el Sentido, Utilidad y 
Efectos de estas Pruebas en el Campo Educativo. 1Universidad de Cádiz y Universidad de Málaga

Antoni Sanz Martín. (2008). La evaluación de los Aprendizajes: Construcción e Instrumento. Universitat de Barcelona. Institut de Ciéncies de L'Educació

Aurora Lacueva. (1997). La evaluación en la Escuela: Una Ayuda Para seguir Aprendiendo. Revista de Facultad de Educación. Brasil

Serrano de Moreno, Stella. (2002). La evaluación del aprendizaje: dimensiones y prácticas innovadoras. Educere, vol. 6, núm. 19, octubre-diciembre, (pp. 247257). Universidad de los Andes. Mérida, Venezuela

Elena Barberà. (2016). Aportaciones de la tecnología a la e-Evaluación. Universidad Oberta de Catalunya. España

José Ma Arribas Estebaranz. Juan Carlos Manrique Arribas y Belén Tabernero Sánchez. (2014). Instrumentos de evaluación utilizados en la formación inicial del profesorado y su coherencia para el desarrollo de competencias profesionales en los estudiantes: visión del alumnado, egresados y profesorado. Universidad de Valladolid y Universidad de Salamanca

Verónica del Carmen Villafaña Rojas. (2015). La importancia de la retroalimentación como parte del proceso de enseñanza-aprendizaje. Instituto Universitario Franco Inglés de México

Velásquez Opazo, Francisco Javier, and Juan Guerrero Núñez. (2012) Procesamiento de datos utilizando una herramienta informática para facilitar la corrección de evaluaciones en clases. Tesis Universidad de Santiago de Chile, 